\title{
Experiments in Stochastic Thermodynamics: Short History and Perspectives
}

\author{
S. Ciliberto \\ Université de Lyon, CNRS, École Normale Supérieure de Lyon, Laboratoire de Physique (UMR5672), \\ 46 Allée d'Italie 69364 Lyon Cedex 07, France
}

(Received 5 April 2017; published 30 June 2017)

\begin{abstract}
We summarize in this article the experiments which have been performed to test the theoretical findings in stochastic thermodynamics such as fluctuation theorem, Jarzynski equality, stochastic entropy, out-ofequilibrium fluctuation dissipation theorem, and the generalized first and second laws. We briefly describe experiments on mechanical oscillators, colloids, biological systems, and electric circuits in which the statistical properties of out-of-equilibrium fluctuations have been measured and characterized using the abovementioned tools. We discuss the main findings and drawbacks. Special emphasis is given to the connection between information and thermodynamics. The perspectives and followup of stochastic thermodynamics in future experiments and in practical applications are also discussed.
\end{abstract}

DOI: 10.1103/PhysRevX.7.021051

Subject Areas: Statistical Physics

\section{INTRODUCTION}

When the size of a system is reduced, the role of fluctuations (either quantum or thermal) increases. Thus, thermodynamic quantities such as internal energy, work, heat, and entropy cannot be characterized only by their mean values, but also their fluctuations and probability distributions become relevant and useful to make predictions on a small system. Let us consider a simple example, such as the motion of a Brownian particle subjected to a constant external force. Because of thermal fluctuations, the work performed on the particle by this force per unit time, i.e., the injected power, fluctuates, and the smaller the force, the larger is the importance of power fluctuations [1-3]. The goal of stochastic thermodynamics is just that of studying the fluctuations of the abovementioned thermodynamic quantities in systems driven out of equilibrium by external forces, temperature differences, and chemical reactions. For this reason, it has received in the past 20 years an increasing interest for its applications in microscopic devices and biological systems and for its connections with information theory [1-3].

In the following we discuss the role of fluctuations in out-of-equilibrium thermal systems when the energies injected or dissipated are smaller than $100 k_{B} T$ ( $k_{B}$ being the Boltzmann constant and $T$ the temperature). This limit is relevant in biological, nano, and micro systems, where fluctuations cannot be neglected. We are interested in knowing the role of these fluctuations on the dynamics and how one can gain some information by measuring them.

Published by the American Physical Society under the terms of the Creative Commons Attribution 4.0 International license. Further distribution of this work must maintain attribution to the author(s) and the published article's title, journal citation, and DOI.
We have already mentioned the Brownian particle driven by an external force, but to clarify the kind of questions that we want to analyze, let us consider another simple example of an out-of-equilibrium system, that is, a thermal conductor whose extremities are connected to two heat baths at different temperatures. The second law of thermodynamics imposes that the mean heat flux flows from the hot to the cold reservoir. However, the second law does not say anything about fluctuations, and in principle one can observe for a short time a heat current in the opposite direction, which corresponds to an instantaneous negative entropy production rate. What is the probability of observing these rare events? The same problem appears in the abovementioned example of the Brownian particle where one can ask, what is the probability that the particles move in the opposite direction of the force? The answer to these questions can be found within the framework of stochastic thermodynamics and fluctuation theorems (FTs) [4-12], which uses statistical mechanics to answer questions related to extremes that are well beyond the mean (i.e., thermodynamics) and well beyond the standard fluctuation theory normally dominated, away from critical points by the central limit theorem. We see that the knowledge of outof-equilibrium fluctuation properties is actually very useful in experiments to extract useful information on equilibrium and out-of-equilibrium properties of a specific system. Typical examples are the Jarzynski and Crooks equalities [13-15], which estimate equilibrium properties starting from nonequilibrium measurements. The measurement of the linear response in out-of-equilibrium systems is another very important aspect. Indeed, the new formulations of the fluctuation dissipation relation (FDR) related to the FT are quite useful for this purpose, because they allow the estimation of the response starting from the measurement of fluctuations of different quantities in nonequilibrium steady states (NESSs) [10,16-21]. Within the context of the 
FDR for out-of-equilibrium states, many studies have been done on the slow relaxation toward equilibrium, such as in aging glasses after a temperature quench [22-24]. It turns out that entropy production plays a unifying role between the FT and the different extended formulations of the FDR for out-of-equilibrium systems.

Another application of stochastic thermodynamics is the study of the efficiency of micro or nano devices and the role of fluctuations in the power production of these systems. This is of course very useful for understanding and measuring the efficiency of molecular motors, which are isothermal and driven by chemical reactions.

It is worth mentioning that the study of stochastic thermodynamics has allowed us to bring more insight to the connection between information and thermodynamics. Specifically, the study of the energy fluctuations in a small system has transformed gedanken experiments, such as the Maxwell's demon, in experiments which may actually be performed thanks to the new technologies such as optical or electrical traps and single electron devices.

Before explaining how the article is organized, it is mandatory to point out that the tools of stochastic thermodynamics have also been applied to study the properties of macroscopic fluctuations in out-of-equilibrium systems. Indeed, the injected and dissipated energies may also fluctuate in macroscopic systems if the dynamics is chaotic. For instance, think of a motor used to stir a fluid strongly. The motor can be driven by imposing a constant velocity. Because of the turbulent motion of the fluid, the power needed to keep the velocity constant fluctuates [25,26]. This simple example shows that fluctuations of the injected and dissipated power may be relevant not only in microscopic but also in macroscopic systems such as hydrodynamic flows [26], granular media [27-30], mechanical systems [31], and more recently on self-propelling particles $[32,33]$. The main difference is that in macroscopic systems fluctuations are produced by the dynamics and are sustained by a constant energy flux, whereas in small systems they are of either thermal or quantum nature. Thus, it is useful to divide the fluctuation in out-of-equilibrium systems into two classes: one where thermal fluctuations play a significant role (thermal systems) and another where the fluctuations are produced by chaotic flows or fluctuating driving forces (athermal chaotic systems). In this article, we focus on thermal systems and only a short discussion on the problems related to the application of stochastic thermodynamics to athermal systems is provided in Sec. IX G.

As the goal of this article is to present several general experimental aspects, we follow an experimentalist approach, and the connection with theory is made on the basis of experimental measurements. Furthermore, we organize the sections in terms of the main topics and tools of stochastic thermodynamics. For this reason, the same experimental apparatus is analyzed in various sections using different theoretical tools We start in Sec. II with an analysis of the experimental results on the energy fluctuations in a harmonic oscillator driven out of equilibrium by an external force. In Sec. III, we describe the properties of FTs, and as illustrative examples we apply it to (a) a harmonic oscillator (linear case) and (b) a Brownian particle confined in a time-dependent double-well potential (nonlinear case). The latter is one of the very few examples where the FT is applied to a highly nonlinear potential, because most of the experiments reported in the literature are performed for linear potentials. In Sec. IV, we discuss the application of the Jarzinsky and Crooks equalities to the harmonic oscillators and to the measure of the free energy of a single molecule. In Sec. VI, we introduce the application of stochastic thermodynamics to the study of the efficiency of micro or nano machines. The contribution of fluctuations to the power produced by these machines is described using the results of a proof of principle experiment. In Sec. VII, we briefly present another relevant aspect of nonequilibrium statistical mechanics: the measurement of the linear response of a system in a nonequilibrium state. We discuss here only the main relevant features without giving any specific example as these FDT aspects have already been discussed in other reviews $[2,3,34]$. In Sec. VIII, the connections between information and thermodynamics is analyzed following two complementary subjects. The first is the energy production by devices controlled by a Maxwell's demon. The second is the minimum energy needed to process one bit of information. Finally, we conclude in Sec. IX, where we describe other useful experimental applications of stochastic thermodynamics. We also discuss the perspectives and the artifacts of these applications.

\section{WORK AND HEAT FLUCTUATIONS IN THE HARMONIC OSCILLATOR}

The choice of discussing the dynamics of the harmonic oscillator is dictated by the fact that it is relevant for many practical applications, such as the measure of the elasticity of nanotubes [35], the dynamics of the tip of an AFM [36], MEMS, and the thermal rheometer that we developed several years ago to study the rheology of complex fluids [37], whose high sensitivity has actually allowed several tests of FT. As the results of these experiments have already been discussed in some detail in several reviews $[3,38]$, we describe here only the main results useful to introduce the experimental activity in stochastic thermodynamics.

\section{A. Experimental setup}

The thermal rheometer is a torsion pendulum whose angular displacement $\theta$ is measured by a very sensitive interferometer. The details of the setup can be found in Refs. [39-43]. A schematic diagram and a picture of the apparatus are shown in Fig. 1. 


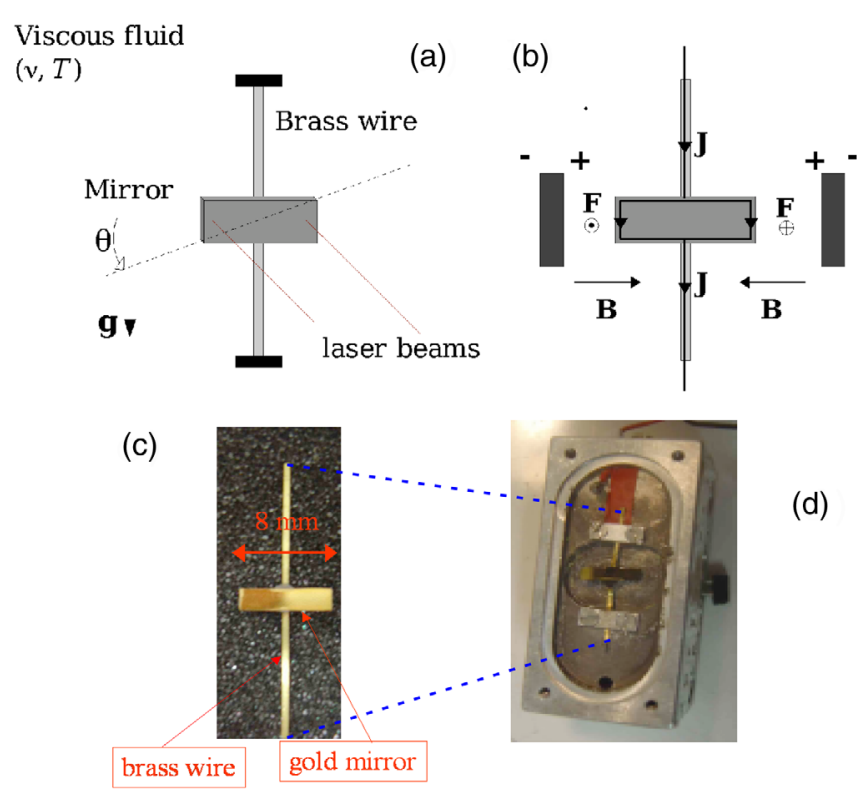

FIG. 1. (a) The torsion pendulum. (b) The magnetostatic forcing. (c) A picture of the pendulum. (d) The cell where the pendulum is installed.

In equilibrium the variance $\delta \theta^{2}$ of the thermal fluctuations of $\theta$ can be obtained from equipartition; i.e., for our pendulum, $\delta \theta=\sqrt{k_{B} T / C} \simeq 2 \mathrm{nrad}$, where $C$ is the torsional stiffness of the pendulum and $T$ is the temperature of the surrounding fluid. The measurement noise is 2 orders of magnitude smaller than thermal fluctuations of the pendulum whose resonance frequency $f_{o}$ is about $217 \mathrm{~Hz}$. A magnetostatic forcing $[38,40,41]$ allows the application of an external torque $M$, useful to excite the pendulum and to drive it out of equilibrium. The typical applied torque is of the order of a few $\mathrm{pN} \mathrm{m}$, and the mean power a few $k_{B} T / s$.

The dynamics of the torsion pendulum can be assimilated to that of a harmonic oscillator damped by the viscosity of the surrounding fluid, whose equation of motion reads

$$
I_{\mathrm{eff}} \frac{d^{2} \theta}{d t^{2}}+\nu \frac{d \theta}{d t}+C \theta=M+\eta
$$

where $I_{\text {eff }}$ is the effective moment of inertia of the pendulum, which includes the inertia of the surrounding fluid as discussed in Ref. [40]. The thermal noise $\eta$ is, in this case, delta correlated in time: $\left\langle\eta(t) \eta\left(t^{\prime}\right)\right\rangle=$ $2 k_{B} T \nu \delta\left(t-t^{\prime}\right)$. However, if the fluid is viscoelastic, the noise $\eta$ is correlated and the process is not Markovian, whereas in the viscous case the process is Markovian. Thus, by changing the quality of the fluid surrounding the pendulum one can tune the Markovian nature of the process. In this review we consider only the experiment in the glycerol-water mixture where the viscoelastic contribution is visible only at very low frequencies and is therefore negligible. This allows a more precise comparison with theoretical predictions often obtained for Markovian processes. (See Ref. [38] for a discussion on this point.)

\section{B. Energy balance}

When the system is driven out of equilibrium by the external deterministic torque $M$ (which is, in general, time dependent), it receives an amount of work, and a fraction of this energy is dissipated into the heat bath. Multiplying Eq. (1) by $\dot{\theta}$ and integrating between $t_{i}$ and $t_{i}+\tau$, one obtains a formulation of the first law of thermodynamics between the two states at time $t_{i}$ and $t_{i}+\tau$ [Eq. (2)]. This formulation was first proposed in Ref. [44] and widely used in other theoretical and experimental works in the context of stochastic thermodynamics [1,2]. The change in internal energy $\Delta U_{\tau}$ of the oscillator over a time $\tau$, starting at a time $t_{i}$, is written as

$$
\Delta U_{\tau}=U\left(t_{i}+\tau\right)-U\left(t_{i}\right)=\tilde{W}_{\tau}-Q_{\tau},
$$

where $\tilde{W}_{\tau}$ is the work done on the system over a time $\tau$ and $Q_{\tau}$ is the dissipated heat. The work $\tilde{W}_{\tau}$ is defined in the classical way,

$$
\tilde{W}_{\tau}=\int_{t_{i}}^{t_{i}+\tau} M\left(t^{\prime}\right) \frac{d \theta\left(t^{\prime}\right)}{d t^{\prime}} d t^{\prime}
$$

and we use a tilde in order to distinguish it from a more general definition often used in stochastic thermodynamics [see Eq. (12) and the discussion at the end of Sec. IVA]. The internal energy is the sum of the potential energy and the kinetic energy:

$$
U(t)=\left\{\frac{1}{2} I_{\mathrm{eff}}\left[\frac{d \theta(t)}{d t}\right]^{2}+\frac{1}{2} C \theta(t)^{2}\right\} .
$$

The heat transfer $Q_{\tau}$ is deduced from Eq. (2). It has two contributions:

$$
Q_{\tau}=\tilde{W}_{\tau}-\Delta U_{\tau}=\int_{t_{i}}^{t_{i}+\tau}\left[\nu\left(\frac{d \theta}{d t^{\prime}}\right)^{2}-\eta\left(t^{\prime}\right) \frac{d \theta}{d t^{\prime}}\right] d t^{\prime},
$$

where the integrals in Eqs. (3) and (5) are performed using the Stratonovich convention.

The first term in Eq. (5) corresponds to the viscous dissipation and is always positive, whereas the second term can be interpreted as the work of the thermal noise, which has a fluctuating sign. The second law of thermodynamics imposes $\left\langle Q_{\tau}\right\rangle$ to be positive. Notice that because of the fluctuations of $\theta$ and $\dot{\theta}$ all the quantities $\tilde{W}_{\tau}, \Delta U_{\tau}$, and $Q_{\tau}$ fluctuate, too. We are interested in characterizing these fluctuations, which are related by Eq. (2), which is a formulation of the first law of thermodynamics between the two states at time $t_{i}$ and $t_{i}+\tau$. Although Eq. (2) has been 

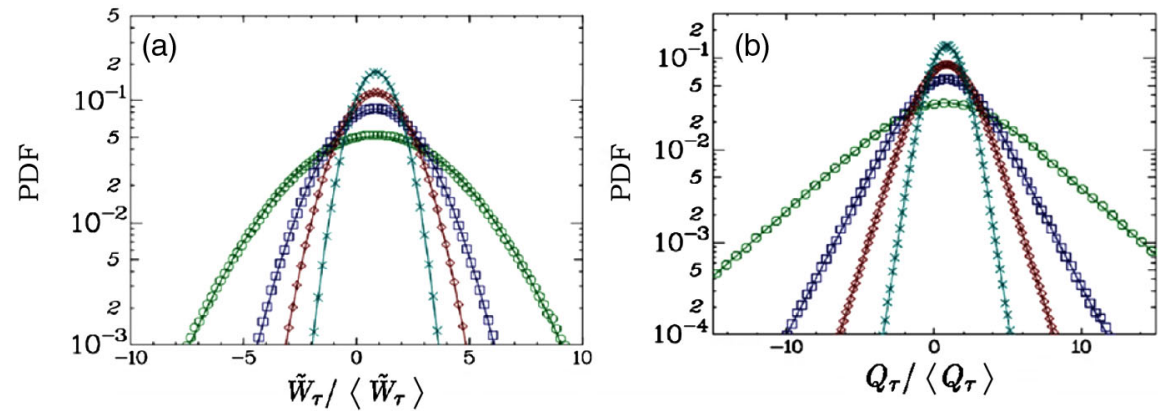

FIG. 2. Sinusoidal forcing. (a) Pdf of $\tilde{W}_{\tau}$. (b) PDF of $Q_{\tau}$ for various $n: n=7$ (॰), $n=15(\square), n=25(\diamond)$, and $n=50(\times)$. The continuous lines are not fits but are analytical predictions obtained from the Langevin dynamics, as discussed in Sec. III C.

obtained for the harmonic oscillators, is indeed a general statement for the energy fluctuations of any system.

\section{Nonequilibrium steady state: Sinusoidal forcing}

We now consider a periodic forcing of amplitude $M_{o}$ and frequency $\omega_{d}$, i.e., $M(t)=M_{o} \sin \left(\omega_{d} t\right)$ [41-43], which is a NESS because all the averages performed on an integer number of the driving periods do not depend on time. This kind of periodic forcing is very common and it has been studied in the case of the first-order Langevin equation [45] and of a two-level system [46] and in a different context for the second-order Langevin equation [47]. Furthermore, this is a very general case, because using Fourier transform any periodical driving can be decomposed in a sum of sinusoidal modes. We explain here the behavior of a single mode. Experiments have been performed at various $M_{o}$ and $\omega_{d}$. We present the results for a particular amplitude and frequency: $M_{o}=0.78 \mathrm{pNm}$ and $\omega_{d} /(2 \pi)=64 \mathrm{~Hz}$.

\section{Work fluctuations}

The work done by $M(t)$ is computed from Eq. (3) on a time $\tau=2 \pi n / \omega_{d}$, i.e., an integer number $n$ of the driving period. $\tilde{W}_{\tau}$ fluctuates and its probability density function (PDF) is plotted in Fig. 2(a) for various $n$. This plot has interesting features. Specifically, work fluctuations are Gaussian for all values of $n$, and $\tilde{W}_{\tau}$ takes negative values as long as $\tau$ is not too large. The probability of having negative values of $\tilde{W}_{\tau}$ decreases when $\tau_{n}$ is increased. There is a finite probability of having negative values of the work; in other words, the system may have an instantaneous negative entropy production rate although the average of the work $\left\langle\tilde{W}_{\tau}\right\rangle$ is, of course, positive $(\langle\cdot\rangle$ stands for ensemble average). In this specific example, $\left\langle\tilde{W}_{\tau}\right\rangle=0.04 n\left(k_{B} T\right)$.

\section{Heat fluctuations}

The dissipated heat $Q_{\tau}$ cannot be directly measured because Eq. (5) requires us to compute the work done by the thermal noise, which is experimentally unmeasurable since $\eta$ is unknown. However, $Q_{\tau}$ can be obtained indirectly from the measure of $\tilde{W}_{\tau}$ and $\Delta U_{\tau}$. We first make some comments on the average values. The average of $\Delta U_{\tau}$ is obviously vanishing because the time $\tau$ is a multiple of the period of the forcing. Therefore, $\left\langle\tilde{W}_{\tau}\right\rangle$ and $\left\langle Q_{\tau}\right\rangle$ are equal, as it must be.

We rescale the work $\tilde{W}_{\tau}$ (the heat $Q_{\tau}$ ) by the average work $\left\langle\tilde{W}_{\tau}\right\rangle$ (the average heat $\left\langle Q_{\tau}\right\rangle$ ) and define $w_{\tau}=$ $\left(\tilde{W}_{\tau} /\left\langle\tilde{W}_{\tau}\right\rangle\right)\left(q_{\tau}=\left(Q_{\tau} /\left\langle Q_{\tau}\right\rangle\right)\right)$. In the present article, $x_{\tau}$ $\left(X_{\tau}\right)$ stands for either $w_{\tau}$ or $q_{\tau}\left(\tilde{W}_{\tau}\right.$ or $\left.Q_{\tau}\right)$.

We compare now the PDF of $w_{\tau}$ and $q_{\tau}$ in Fig. 2. The PDFs of heat fluctuations $q_{\tau}$ have exponential tails [Fig. 2(b)]. It is interesting to stress that although the two variables $\tilde{W}_{\tau}$ and $Q_{\tau}$ have the same mean values, they have a very different PDF. The PDFs of $w_{\tau}$ are Gaussian, whereas those of $q_{\tau}$ are exponential. On a first approximation, the PDFs of $q_{\tau}$ are the convolution of a Gaussian (the PDF of $\tilde{W}_{\tau}$ ) and exponential (the PDF of $\Delta U_{\tau}$ ) $[38,43]$. In Fig. 2, the continuous lines are analytical predictions obtained from the Langevin dynamics with no adjustable parameter (see Sec. III C).

\section{FLUCTUATION THEOREMS}

In the previous section, we see that both $\tilde{W}_{\tau}$ and $Q_{\tau}$ present negative values; i.e., the second law is verified only on average, but the entropy production can have instantaneously negative values. The probabilities of getting positive and negative entropy production are quantitatively related in nonequilibrium systems by the fluctuation theorems [4-6],

There are two classes of FTs. The stationary state fluctuation theorem (SSFT) considers a nonequilibrium steady state. The transient fluctuation theorem (TFT) describes transient nonequilibrium states where $\tau$ measures the time since the system left the equilibrium state. A fluctuation relation examines the symmetry around 0 of the probability density function $p\left(x_{\tau}\right)$ of a quantity $x_{\tau}$, as defined in the previous section. It compares the probability of having a positive event $\left(x_{\tau}=+x\right)$ versus the probability of having a negative event $\left(x_{\tau}=-x\right)$. We quantify the FT using a function (symmetry function): 


$$
\operatorname{Sym}\left(x_{\tau}\right)=\frac{k_{B} T}{\left\langle X_{\tau}\right\rangle} \ln \left(\frac{p\left(x_{\tau}=+x\right)}{p\left(x_{\tau}=-x\right)}\right) .
$$

The TFT states that the symmetry function is linear with $x_{\tau}$ for any values of the time integration $\tau$ and the proportionality coefficient is equal to 1 for any value of $\tau$ :

$$
\operatorname{Sym}\left(x_{\tau}\right)=x_{\tau}, \quad \forall x_{\tau}, \quad \forall \tau .
$$

Contrary to TFT, the SSFT holds only in the limit of infinite time $(\tau)$ :

$$
\lim _{\tau \rightarrow \infty} \operatorname{Sym}\left(x_{\tau}\right)=x_{\tau} .
$$

In the following, we assume linearity at finite time $\tau$ $[48,49]$ and use the following general expression:

$$
\operatorname{Sym}\left(x_{\tau}\right)=\Sigma_{x}(\tau) x_{\tau},
$$

where for SSFT $\Sigma_{x}(\tau)$ takes into account the finite-time corrections and $\lim _{\tau \rightarrow \infty} \Sigma_{x}(\tau)=1$, whereas $\Sigma_{x}(\tau)=1, \forall \tau$ for TFT.

However, these claims are not universal because they depend on the kind of $x_{\tau}$ that is used. Specifically, we see in the next sections that the results are not exactly the same if $X_{\tau}$ is replaced by any one of $\tilde{W}_{\tau}, Q_{\tau}$, and the total entropy $[11,12]$. Furthermore, the definitions given in this section are appropriate for stochastic systems, and the differences between stochastic and chaotic systems are not addressed in this review. A discussion on this point can be found in Ref. [38].

\section{A. FTs for $\tilde{W}_{\tau}$ and $Q_{\tau}$ measured in the harmonic oscillator}

The questions we ask are whether for finite time FTs are satisfied for either $x_{\tau}=w_{\tau}$ or $x_{\tau}=q_{\tau}$ and what are the finite-time corrections? As a first step, we test the correction to the proportionality between the symmetry function
$\operatorname{Sym}\left(x_{\tau}\right)$ and $x_{\tau}$. In the region where the symmetry function is linear with $x_{\tau}$, we define the slope $\Sigma_{x}(\tau)$, i.e., $\operatorname{Sym}\left(x_{\tau}\right)=\Sigma_{x}(\tau) x_{\tau}$. As a second step, we measure finitetime corrections to the value $\Sigma_{x}(\tau)=1$, which is the asymptotic value expected from FTs.

In this article, we focus on the SSFT applied to the experimental results of Sec. II C and to other examples. The applications of TFT are not presented in this section, but we discuss them in Sec. IV B, and interested readers may find more details in Ref. [43].

From the PDFs of $w_{\tau}$ and $q_{\tau}$ plotted in Fig. 2, we compute the symmetry functions defined in Eq. (6). The symmetry functions $\operatorname{Sym}\left(w_{\tau}\right)$ are plotted in Fig. 3(a) as a function of $w_{\tau}$. They are linear in $w_{\tau}$. The slope $\Sigma_{w}(n)$ is not equal to 1 for all $n$, but there is a correction at finite time. Nevertheless, $\Sigma_{w}(n)$ tends to 1 for large $n$. Thus, SSFT is satisfied for $\tilde{W}_{\tau}$ and for a sinusoidal forcing. The convergence is very slow and we have to wait a large number of periods of forcing for the slope to be 1 (after 30 periods, the slope is still 0.9). This behavior is independent of the amplitude of the forcing $M_{o}$ and consequently of the mean value of the work $\left\langle\tilde{W}_{\tau}\right\rangle$, which, as explained in Ref. [38], changes only the time needed to observe a negative event. The system satisfies the SSFT for all forcing frequencies $\omega_{d}$, but finite-time corrections depend on $\omega_{d}$ [43].

We now analyze the PDF of $q_{\tau}$ [Fig. 2(b)] and we compute the symmetry functions $\operatorname{Sym}\left(q_{\tau}\right)$ of $q_{\tau}$ plotted in Fig. 3(b) for different values of $n$. They are clearly very different from those of $w_{n}$ plotted in Fig. 3(a). For $\operatorname{Sym}\left(q_{\tau}\right)$ three different regions appear.

(I) For large fluctuations $q_{n}, \operatorname{Sym}\left(q_{\tau}\right)$ equals 2 . When $\tau$ tends to infinity, this region spans from $q_{n}=3$ to infinity.

(II) For small fluctuations $q_{n}, \operatorname{Sym}\left(q_{n}\right)$ is a linear function of $q_{n}$. We then define $\Sigma_{q}(n)$ as the slope of the function $\operatorname{Sym}\left(q_{n}\right)$, i.e., $\operatorname{Sym}\left(q_{n}\right)=\Sigma_{q}(n) q_{n}$. We have measured [43] that $\Sigma_{q}(n)=\Sigma_{w}(n)$ for all the values of $n$; i.e., finite-time corrections are the same for heat and work. Thus, $\Sigma_{q}(n)$ tends to 1 when $\tau$ is increased and SSFT

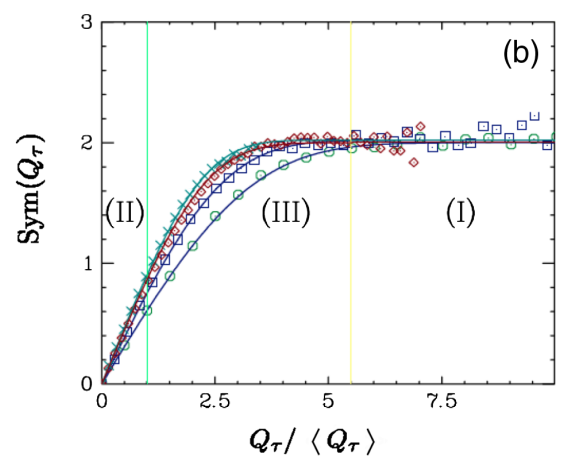

FIG. 3. Sinusoidal forcing. Symmetry functions for SSFT. (a) Symmetry functions $\operatorname{Sym}\left(w_{\tau}\right)$ plotted as a function of $w_{\tau}$ for various $n$ : $n=7$ (circle), $n=15$ (square), $n=25$ (diamond), and $n=50$ (times). For all $n$, the dependence of Sym $\left(w_{\tau}\right)$ on $w_{\tau}$ is linear, with slope $\Sigma_{w}(\tau)$. (b) Symmetry functions $\operatorname{Sym}\left(q_{\tau}\right)$ plotted as a function of $q_{\tau}$ for various $n$. The dependence of $\operatorname{Sym}\left(q_{\tau}\right)$ on $q_{\tau}$ is linear only for $q_{\tau}<1$. Continuous lines are theoretical predictions. 
holds in this region II, which spans from $q_{n}=0$ up to $q_{n}=1$ for large $\tau$. This effect was discussed for the first time in Refs. [48,49].

(III) A smooth connection between the two behaviors.

These regions define the fluctuation relation from the heat dissipated by the oscillator. The limit for large $\tau$ of the symmetry function $\operatorname{Sym}\left(q_{\tau}\right)$ is rather delicate and it is discussed in Ref. [43].

The conclusion of this experimental analysis is that SSFT holds for work for any value of $w_{\tau}$, whereas for heat it holds only for $q_{\tau}<1$. The finite-time corrections to FTs, described by $1-\Sigma$, are not universal. They are the same for both $w_{\tau}$ and $q_{\tau}$, but they depend on the driving frequency and on the kind of driving force Ref. [38,43].

These kinds of measurements are important because they allow us to test complex theoretical concepts on relatively simple systems in order to apply them to more complex cases. Furthermore, the experimental analysis on model systems allows us to check the theoretical hypothesis made in order to prove the theorems. For example, one of these hypotheses is that the properties of the heat bath are not modified by the forcing. This hypothesis can be precisely checked in the experiments.

\section{B. Trajectory-dependent entropy and the total entropy}

In the same way of $\tilde{W}_{\tau}$ and $Q_{\tau}$, the entropy production rate can also be defined at the trajectory level. The trajectory-dependent entropy difference $\delta s_{\tau}(t)$ is defined as $\delta s_{\tau}(t)=-\log [P(\vec{r}(t+\tau), \lambda) / P(\vec{r}(t), \lambda)]$, where $P(\vec{r}(t), \lambda)$ is the probability of finding the system in the position $\vec{r}(t)$ of the phase space at a value $\lambda$ of the control parameter. Thus, the total entropy difference on the time $\tau$ is $\Delta S_{\mathrm{tot}}(t, \tau)=\delta s_{\tau}(t)+Q_{\tau}(t) / T[11,12]$, i.e., the sum of the trajectory-dependent entropy and of the entropy change in the reservoir due to energy flow. The mean total entropy difference is equal to the entropy production rate; i.e., $\left\langle\Delta S_{\text {tot }}(t, \tau)\right\rangle=\left\langle Q_{\tau}(t) / T\right\rangle$. Furthermore, $\Delta S_{\text {tot }}(t, \tau)$ fully characterizes the out-of-equilibrium dynamics as it is rigorously zero, both in average and fluctuations. The fluctuations of this quantity impose several constrains on the time reversibility, which is a central result of stochastic thermodynamics $[2,11,15,50]$ and which has been tested experimentally [51]. The FT for the $\Delta S_{\text {tot }}$ in a SSFT implies $\Sigma(\tau)=1$ for any $\tau$; i.e., the FT does not have an asymptotic validity but is valid for any $\tau$. This is certainly a useful property in experiment because one does not have to look for very long asymptotic behavior. However, the calculation of $S_{\text {tot }}$ in experiment is not easy and a lot of care must be used in order to correctly estimate this quantity [38,52]. We do not discuss here the experimental analysis performed on electric circuits and harmonic oscillators, but an example of the evaluation of $\Delta S_{\text {tot }}(t, \tau)$ is given in Sec. V. For further information, the interested reader can look at the abovementioned references and Ref. [53], where a discussion on the different quantities for SSFT and TFT can be found.

Furthermore, in order to avoid the complexity of computing $\Delta S_{\text {tot }}$ from individual trajectories, another quantity, which satisfies a SSFT for any $\tau$, has been proposed in Ref. [54]. This quantity is the joint probability $P\left(\tilde{W}_{\tau}, J_{\tau}\right)$ of the work and of the energy currents in the system. Although its measure might be difficult, it is by far easier than the trajectory-dependent entropy. However, this method, although very powerful, has never been tested on experimental data, but it will certainly be useful to try.

\section{Comparison with theory}

The experimental analysis described in Sec. III A allows a very precise comparison with theoretical predictions using the Langevin equation [Eq. (1)] and using two experimental observations: (a) the properties of the heat bath are not modified by the driving and (b) the fluctuations of the $\tilde{W}_{\tau}$ are Gaussian (see also Ref. [55], where it is shown that in Langevin dynamics $\tilde{W}_{\tau}$ has a Gaussian distribution for any kind of deterministic driving force if the properties of the bath are not modified by the driving and the potential is harmonic). The observation in point (a) is extremely important because it is always assumed to be true in all the theoretical analysis. In Ref. [43], this point has been precisely checked. Using these experimental observations one can compute the PDF of $q_{\tau}$ and the finite-time corrections $\Sigma(\tau)$ to SSFT (see Ref. [43]). The continuous lines in Figs. 2 and 3 are not fit but analytical predictions, with no adjustable parameters, derived from the Langevin dynamics of Eq. (1) (see Ref. [43] for more details).

\section{Nonlinear case: Stochastic resonance}

In the harmonic oscillator described in the previous section, the only nonlinearities, which might appear, are those related to the elasticity of the torsion wire. However, to reach this nonlinear regime, the system has to be forced to such a high level that thermal fluctuations become negligible. Thus, in order to study the nonlinear effects we change the experiment and we measure the fluctuations of a Brownian particle trapped in a nonlinear potential produced by two laser beams, as shown in Fig. 4 [56]. It is very well known that a particle of small radius $R \simeq 2 \mu \mathrm{m}$ can be trapped by a focused laser beam, which produces a harmonic potential, thereby confining the Brownian particle motion to the potential well. When two laser beams are focused at a distance $D \simeq R$, as shown in Fig. 4(a), the particle has two equilibrium positions, i.e., the foci of the two beams. Thermal fluctuations allow the particle to hop from one to the other. The particle feels an equilibrium potential $U_{0}(x)=a x^{4}-b x^{2}-d x$, shown in Fig. 4(b), where $a, b$, and $d$ are determined by the laser intensity and by the distance of the two focal points. This potential has been computed from the measured equilibrium 

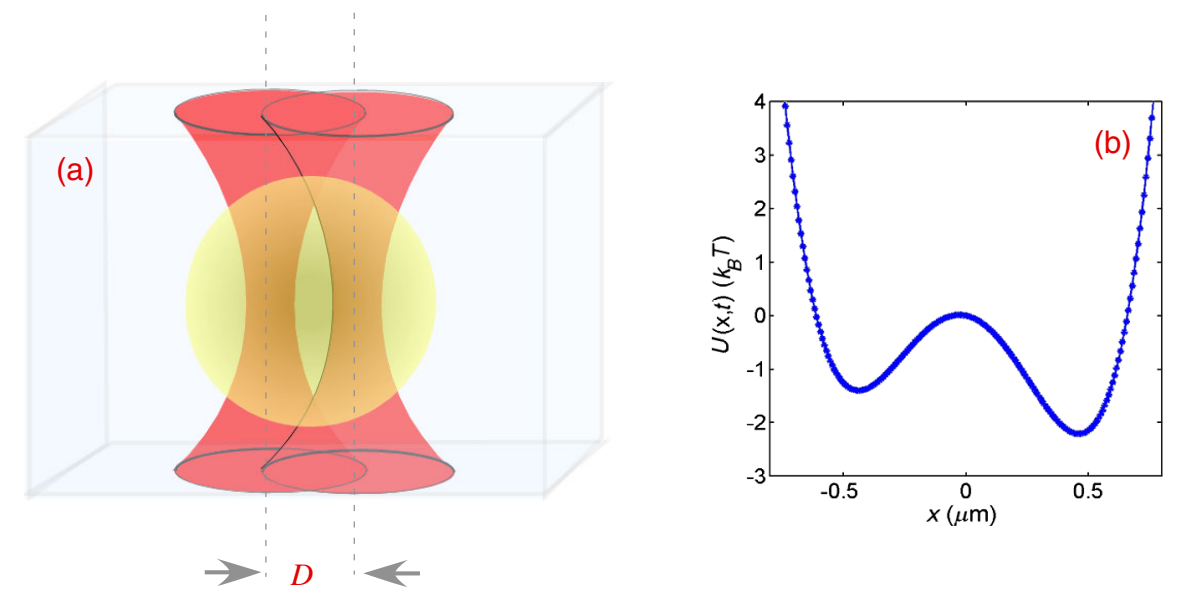

FIG. 4. (a) Drawing of the polystyrene particle trapped by two laser beams whose axis distance is about the radius of the bead. (b) Potential felt by the bead trapped by the two laser beams. The barrier height between the two wells is about $2 k_{B} T$.

distribution of the particle, $P(x) \propto \exp \left[U_{0}(x)\right]$ (see Ref. [56] for more experimental details). To drive the system out of equilibrium we periodically modulate the intensity of the two beams at low frequency $\omega$. Thus, the potential felt by the bead has the following profile: $U(x, t)=U_{0}(x)+U_{p}(x, t)=U_{0}+c x \sin (\omega t)$.

The $x$ position of the particle can be described by an overdamped Langevin equation:

$$
\nu \frac{d x}{d t}=-\frac{\partial U(x, t)}{\partial x}+\eta
$$

with $\nu$ the friction coefficient and $\eta$ the thermal noise delta correlated in time. When $c \neq 0$, the particle can experience a stochastic resonance [57-59], when the forcing frequency is close to the Kramers rate [56]. As already done in the case of the harmonic oscillator, one can compute the work

$$
\tilde{W}_{\tau}=\int_{t}^{t+\tau} f\left(t^{\prime}\right) \dot{x}\left(t^{\prime}\right) d t^{\prime}
$$

of the external force $f(t)=-c \sin (2 \pi f t)$ on the time interval $[t, t+\tau]$, where $\tau=(2 \pi n / \omega)$ is a multiple of the forcing period [56].

We consider the PDF $P\left(\tilde{W}_{\tau}\right)$, which is plotted in Fig. 5(a). Notice that for small $n$ the distributions are double peaked and very complex. They tend to a Gaussian for large $n$ [inset of Fig. 5(a)]. In Fig. 5(b) we plot the normalized symmetry function of $\tilde{W}_{\tau}$. We can see that the curves are close to the line of slope one. For high values of work, the dispersion of the data increases due to the lack of events. The slope tends toward 1 as expected by the SSFT. It is remarkable that straight lines are obtained even for $n$ close to 1 , where the distribution presents a very complex and unusual shape [Fig. 5(a)]. The very fast convergence to the asymptotic value of the SSFT is quite striking in this example. We do not show here $S\left(Q_{\tau}\right)$ as the behavior is quite similar to that of the harmonic oscillator (Sec. III A) although the PDFs are more complex [56]. The measurement are in full agreement with a realistic model based on the Fokker-Planck equations where the measured values of $U(x, t)$ have been inserted [60]. This example shows the
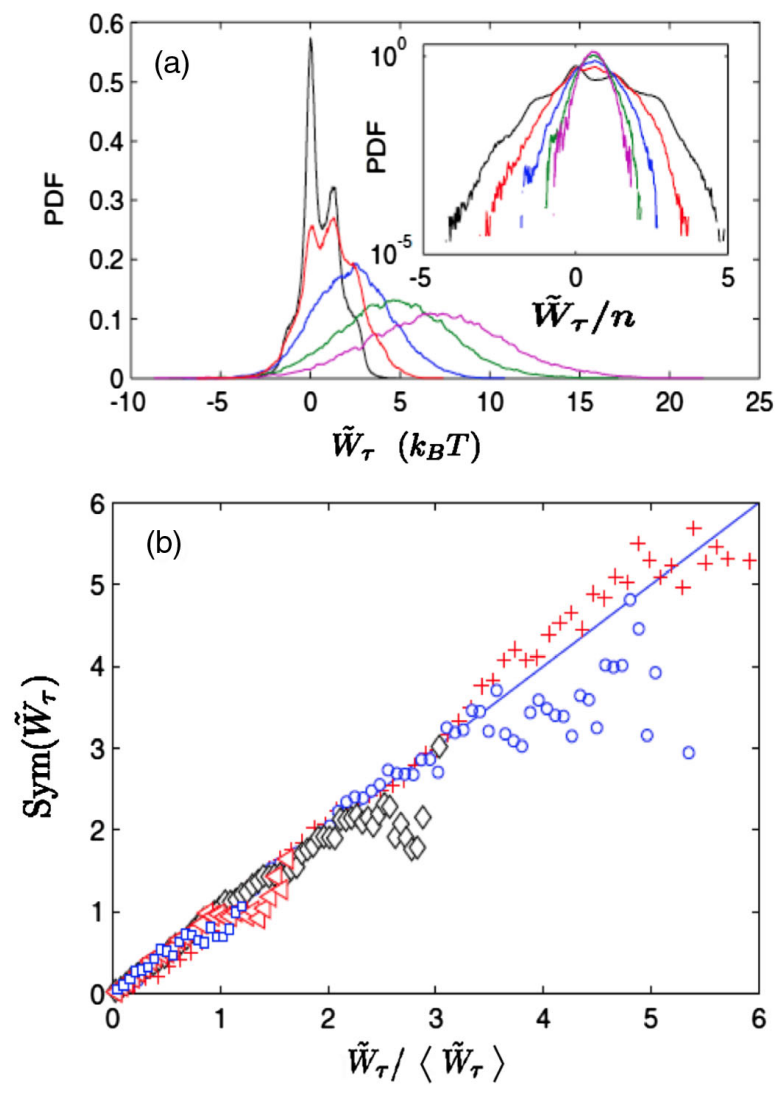

FIG. 5. (a) Distribution of classical work $\tilde{W}_{\tau}$ for different numbers of period $n=1,2,4,8$, and 12 ( $f=0.25 \mathrm{~Hz}$ ). Inset: Same data in lin-log. (b) Normalized symmetry function as function of the normalized work for $n=1$ (plus), 2 (circle), 4 (diamond), 8 (triangle), and 12 (square). 
application of FT in a nonlinear case where the distributions are strongly non-Gaussian. Other examples of application of FT to nonlinear potentials can be found in Ref. [61].

\section{ESTIMATE THE FREE-ENERGY DIFFERENCE FROM WORK FLUCTUATIONS}

In 1997 [13,14] Jarzynski derived an equality which relates the free-energy difference of a system in contact with a heat reservoir to the PDF of the work performed on the system to drive it from $A$ to $B$ along any path $\gamma$ in the system parameter space.

\section{A. Jarzynski equality}

Specifically, when a system parameter $\lambda$ is varied from time $t=0$ to $t=t_{s}$, Jarzynski defines for one realization of the "switching process" from $A$ to $B$ the work performed on the system as

$$
W=\int_{0}^{t_{s}} \dot{\lambda} \frac{\partial H_{\lambda}[z(t)]}{\partial \lambda} d t,
$$

where $z$ denotes the phase-space point of the system and $H_{\lambda}$ its $\lambda$ parametrized Hamiltonian. One can consider an ensemble of realizations of this switching process with initial conditions all starting in the same initial equilibrium state. Then $W$ may be computed for each trajectory in the ensemble. The Jarzynski equality states that $[13,14]$

$$
\exp (-\beta \Delta F)=\langle\exp (-\beta W)\rangle,
$$

where $\langle\cdot\rangle$ denotes the ensemble average and $\beta^{-1}=k_{B} T$ with $k_{B}$ the Boltzmann constant and $T$ the temperature. In other words, $\left\langle\exp \left[-\beta W_{\text {diss }}\right]\right\rangle=1$, since we can always write $W=\Delta F+W_{\text {diss }}$, where $W_{\text {diss }}$ is the dissipated work. Thus, it is easy to see that there must exist some paths $\gamma$ such that $W_{\text {diss }} \leq 0$. Moreover, the inequality $\langle\exp x\rangle \geq$ $\exp \langle x\rangle$ allows us to recover the second principle, namely, $\left\langle W_{\text {diss }}\right\rangle \geq 0$, i.e., $\langle W\rangle \geq \Delta F$. If the probability distribution of the work is Gaussian, $P(W) \propto \exp \left[-\left([W-\langle W\rangle]^{2} / 2 \sigma_{W}^{2}\right)\right]$, then Eq. (13) leads to

$$
\Delta F=\langle W\rangle-\frac{\beta \sigma_{W}^{2}}{2},
$$

i.e., the dissipate energy $\left\langle W_{\text {diss }}\right\rangle=\left(\beta \sigma_{W}^{2} / 2\right)>0$.

From an experimental point of view the Jarzynski equality [Eq. (13)] is quite useful because there is no restriction on the choice of the path $\gamma$. Furthermore, the other big advantage is that only the initial state has to be an equilibrium state. Indeed even if the system is not in equilibrium at time $t_{s}$, one obtains the value of $\Delta F\left(\lambda\left(t_{s}\right)\right)$, i.e., the value of the free energy that the system would have at equilibrium for the value of the control parameter $\lambda=\lambda\left(t_{s}\right)$ [62]. Finally, we stress that the definition of work given in Eq. (12) is more general than the classical definition used in Eqs. (3) and (11).
Specifically, in the experiment described in Sec, III D, $\lambda$ is the external force and $W=[f(t) x(t)]_{t}^{t+\tau}-\tilde{W}_{\tau}$. Thus, in an experiment in order to get the right value of the free energy using Eq. (13), one has to clearly identify the control parameter $\lambda$, which drives the system, and the definition of Eq. (3) must be used to estimate $W$ (see Ref. [40] for a discussion on this point).

\section{B. Crooks relation}

This relation is related to the Jarzynski equality and it gives useful and complementary information on the dissipated work. Crooks considers the forward work $W_{f}$ to drive the system from $A$ to $B$ and the backward work $W_{b}$ to drive it from $B$ to $A$ [Eq. (3) is used for both $W_{f}$ and $W_{b}$ ]. If the work PDFs during the forward and backward processes are $P_{f}(W)$ and $P_{b}(W)$, one has [15]

$$
\frac{P_{f}(W)}{P_{b}(-W)}=\exp (\beta[W-\Delta F])=\exp \left[\beta W_{\text {diss }}\right] .
$$

Notice that the TFT defined in Sec. III is a special case of Eq. (15), for which $A \equiv B$ and the backward and forward protocols are the same.

A simple calculation leads from Eq. (15) to Eq. (13). However, from an experimental point of view, Eq. (15) is extremely useful because one immediately sees that the crossing point of the two PDFs, that is, the point where $P_{f}(W)=P_{b}(-W)$, is precisely $\Delta F$. Thus, one has another mean to check the computed free energy by looking at the PDFs crossing point $W_{\times}$. The draw back is that both states $A$ and $B$ have to be equilibrium states. A very interesting and extended review on the Jarezynski and Crooks relations can be found in Ref. [63].

\section{Applications of Jarzynski and Crooks equalities}

The Jarzynski equality has been tested for the first time in a single-molecule experiment [64]. Here, we follow a more pedagogical description by discussing first the application to the harmonic oscillator and then to a singlemolecule experiment in Sec. IV C 2.

\section{Harmonic oscillator}

As a simple example we apply the Jarzynski equality to the harmonic oscillator described in Sec. II. The oscillator is driven from a state $A(M=0)$ and to a state $B$ (where $M=M_{o} \neq 0$ ) by the external tork $M(t)=M_{o} t / t_{s}$ (forward transformation). The backward transformation is instead $M(t)=M_{o}-M_{o} t / t_{s}$. The switching time $t_{s}$ is varied in order to probe either the reversible (or quasistatic) paths $\left(t_{s} \gg \tau_{\text {relax }}\right)$ or the irreversible ones $\left(t_{s} \lesssim \tau_{\text {relax }}\right)$, where $\tau_{\text {relax }}$ is the harmonic oscillator relaxation time. We apply a torque which is a sequence of linear increasing or decreasing ramps and plateaus. The latter are necessary to relax the system in equilibrium before starting a new 

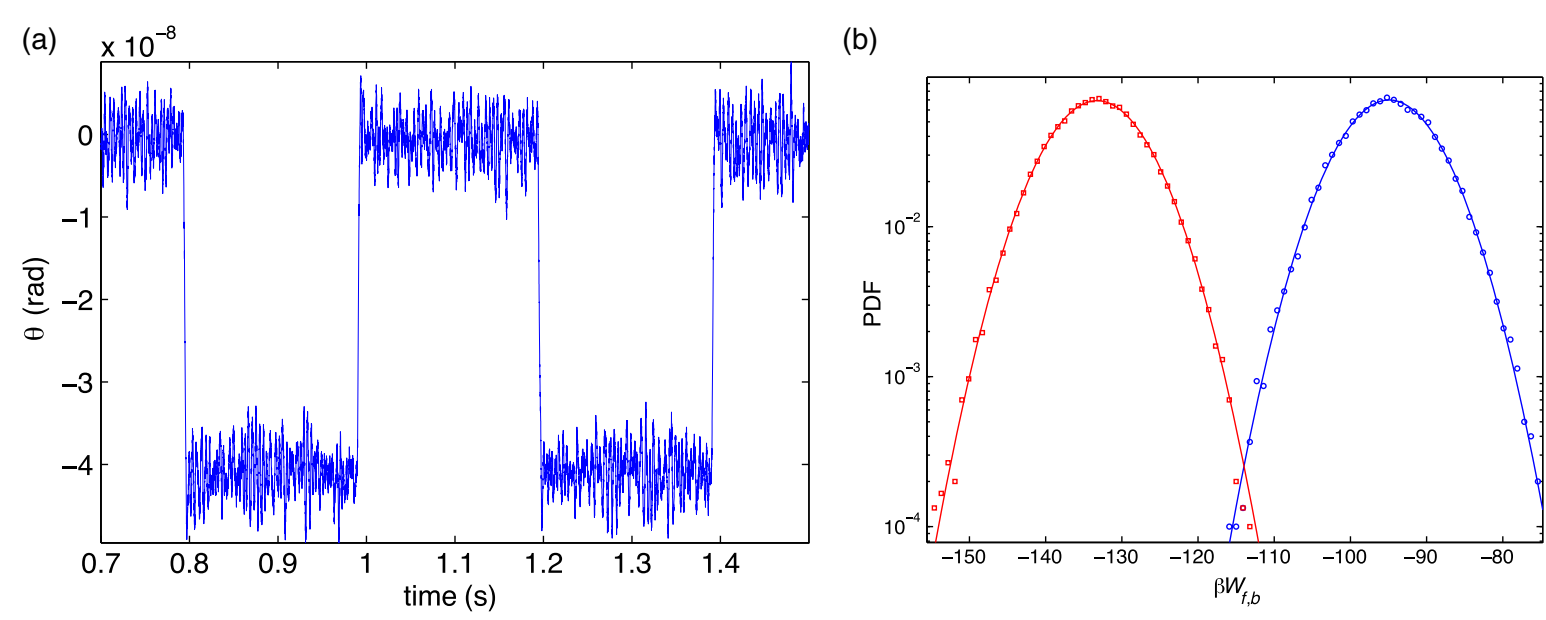

FIG. 6. Free-energy measure in the harmonic oscillator. (a) Time evolution of $\theta(t)$ under a periodic torque $M$ which drives the oscillator from $A(\theta=0)$ to $B\left[\theta\left(t_{s}\right)=4.1 \mathrm{nrad}\right]$ and vice versa. In this specific case the stiffness is $C \simeq 5.5 \mathrm{Nm}$, the transition time is $t_{s} \simeq 0.1 \tau_{\text {relax }}$, and $M_{o}=22.4 \mathrm{Nm} \mathrm{rad}^{-1}$. (b) Probability distribution functions of the work for the forward (blue curve) and backward (red curve) transformation. The crossing point of the two PDFs determines the value of $\Delta F_{A, B}$ [see Eq. (15)]. The crossing point is at $W=-112 k_{B} T$, which is within experimental errors of the expected theoretical $\Delta_{F} \simeq-110 k_{B} T$ (see text).

transformation. This periodic driving produces a sequence of direct $A-B$ paths and reversed ones, $B-A$, of $\theta(t)$, whose time dependence is plotted in Fig. 6(a). The plotted dynamics corresponds to quite irreversible transformation as $t_{s} \ll \tau_{\text {relax }}$; specifically, $t_{s} / \tau_{\text {relax }} \simeq 0.1$. The other relevant parameters of the experiment are $M_{o}=22.4 \mathrm{pN}$ and the stiffness $C=5.5 \times 10^{-4} \mathrm{Nm} \mathrm{rad}^{-1}$. The work $W$ can be computed in each of the reverse or direct paths using Eq. (12), and the $P_{f}(W)$ and $P_{b}(W)$ can be constructed. The result is shown in Fig. 6(b), where the probability distribution functions are plotted. We see that $P_{f}(W)$ and $P_{b}(W)$ cross at about $-112 k_{B} T$, which is the measured value of the $\Delta F$ computed from Eq. (15). Let us compare the measured value with the theoretical estimate. In this specific case of the harmonic oscillator, as the temperature is the same in states $A$ and $B$, the free-energy difference of the oscillator alone is $\Delta F_{0}=\Delta U=\left[\frac{1}{2} C \theta^{2}\right]_{A}^{B}=\left[\left(M_{o}^{2} / 2 C\right)\right]_{A}^{B}$. However, the Jarzynski [Eq. (13)] and Crooks [Eq. (15)] relations compute the free-energy difference of the system (the harmonic oscillator) plus the driving, i.e., $\Delta F=\Delta F_{0}-$ $\left[\left(M^{2} / C\right)\right]_{A}^{B}$, which in this case gives $\Delta F=-\Delta F_{0}$. From the values of the experimental parameters, one gets $\Delta F \simeq 110 k_{B} T$, which is very close to the experimental value. This example shows that indeed using Eqs. (13) and (15) it is possible to obtain the values of the free-energy difference between two states even by doing a very fast transformation with $t_{s} \ll \tau_{\text {relax }}$. However, the applications of Eqs. (13) and (15) to the experiments may present several problems, which are discussed in detail in Ref. [40].

\section{Measure of the free-energy difference of a single molecule}

Jarzynsky and Crooks relations turn out to be a useful tool to measure the free energy of a single molecule
[64-77]. Let us summarize a typical example extracted from Ref. [65], where Eq. (15) has been used to measure the $\Delta F$ between the folded and unfolded states of a DNA hairpin. In Fig. 7(a), a schematic diagram of the experiment is depicted, and the typical structure of the DNA hairpin is shown in Fig. 7(b). In this experiment the DNA hairpin is attached to two beads via double-stranded DNA handles. As these two handles are much stiffer than the hairpin, they do not deform during the stretching cycles that are performed in the following way. The bottom bead is kept by a micropipette, whereas an optical trap captures the top bead. A piezoelectric actuator controls the position of the bottom bead, which, when moved along the vertical axis, stretches the DNA. The difference in positions of the bottom and top beads gives the end-to-end length of the molecule. The optical trap is used to measure the force exerted by the stretched DNA on the top bead. By driving the piezoelectric actuator, the molecule is repeatedly subjected to unfold-refold cycles. Every pulling cycle consists of a stretching process (hereafter referred to as $S$ ) and a releasing (hereafter referred to as $R$ ) process. In the stretching part of the cycle the molecule is stretched from a minimum value of the force $\left(f_{\min } \simeq 10 \mathrm{pN}\right)$, so small that the hairpin is always folded, up to a maximum value of the force $\left(f_{\max } \simeq 20 \mathrm{pN}\right)$, so large that the hairpin is always unfolded. During the releasing part of the cycle the force is decreased from $f_{\max }$ back to $f_{\min }$ at the same rate of the loading cycle, which is the same protocol used for the abovementioned example of the harmonic oscillators. As the stretching force and the displacement of the bead are independently measured, the work can be computed using Eq. (12) at each cycle $S$ and $R$. The corresponding $P_{S}(W)$ and $P_{R}(W)$ histograms can be computed too. The experiment has been repeated for 


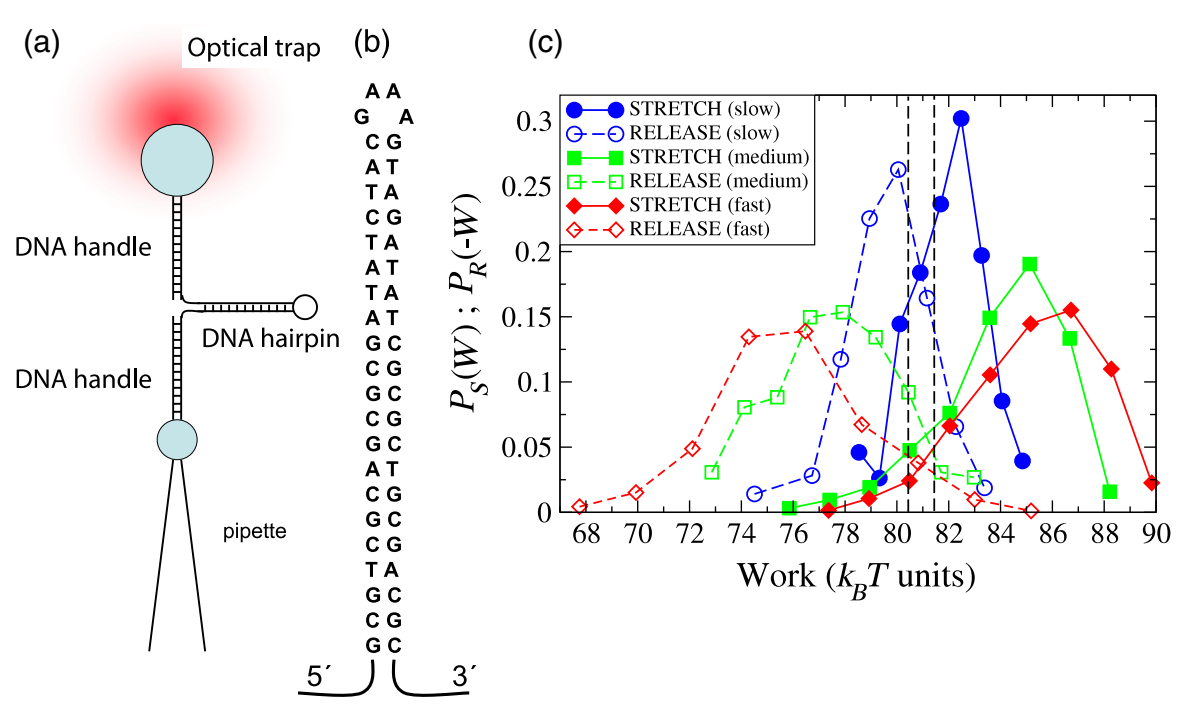

FIG. 7. (a) Experimental setup. The DNA hairpin whose sequence is shown in (b) is attached to two beads. The bottom bead is kept by a micropipette and top bead is captured by an optical trap. The drawing is not to scale; the diameter of the beads is around 3000 nm, much greater than the 20-nm length of the DNA. (b) DNA hairpin sequence. Labels 5 and 3 indicate the polarity of the phosphate chain of the hairpin. (c) Typical work distributions for three different loading rates: $1 \mathrm{pN} / \mathrm{s}$ (slow, blue), $4.88 \mathrm{pN} / \mathrm{s}$ (medium, green), $14.9 \mathrm{pN} / \mathrm{s}$ (fast, red). The vertical lines show the range of the estimated experimental errors for the value of $\Delta F$ (adapted from Ref. [65]).

different pulling-releasing speeds, and the results for three speeds are shown in Fig. 7(c). We see that when the speed is increased the difference between the mean works in the $S$ and $R$ protocols increases. However, the remarkable fact is that the $P_{S}(W)$ and $P_{R}(W)$ cross, within experimental errors at the same value of $W$ independently of the pulling speed, showing the validity of Eq. (15). As already explained, the crossing point gives the value of the free-energy difference between the folded and unfolded states.

\section{Short discussion on applications of Jarzynski and Crooks relations}

The examples in Secs. IV C 1 and IVC 2 show the power of Jarzynski and Crooks relations which are a very useful tool to estimate the free-energy differences of micro and nano systems where the role of fluctuation is very important.

It is worth mentioning that there is a large amount of work on this topic performed by the biophysics and chemistry communities. The estimation of the protein-folding landscapes is an important application, which remains one of the main interests despite many years of investigation; useful examples can be found in Refs. [73,74].

Furthermore, using extensions to the basic results of Jarzynski [68], the works in Refs. [72,75,76] collectively show that nonequilibrium measurements give the most precise reconstructions, to date, of free-energy landscapes for single molecules (DNA hairpins).

The reader might also be interested in a recent extension of these relations by Camunas-Solder et al. [77], who have shown that fluctuation relations can be used for much more than estimating free-energy differences. They study ligand binding and use single-molecule force spectroscopy to measure binding energies, selectivity, and allostery of nucleic acids.

Finally, useful extensions and generalizations of the Jarzynski equality that allow the study of the transition between two nonequilibrium steady states have been derived in Ref. [78] and checked experimentally in Ref. [79].

\section{TWO HEAT BATHS}

In Secs. II, III D, and IV, we discuss systems in contact with a single heat bath, which, within the context of stochastic thermodynamics, are the most studied cases both experimentally and theoretically [2,3]. Conversely, systems, driven out of equilibrium by a temperature gradient, in which the energy exchanges are produced only by the thermal noise, have been analyzed mainly in theoretical models [47,80-90]. This problem has been studied only in a few very recent experiments [91-95], because of the intrinsic difficulties of dealing with large temperature differences in small systems.

In order to illustrate the main properties of the energy fluxes in these systems driven out of equilibrium by a temperature gradient, we summarize in this section the main results of Refs. [91,92]. These two articles analyze both experimentally an theoretically the statistical properties of the energy exchanged between two conductors kept at different temperature and coupled by the electric thermal noise. 


\section{A. Two electric circuits interacting via a conservative coupling}

\section{Experimental setup and stochastic variables}

The experimental setup is sketched in Fig. 8(a). It is constituted by two resistances $R_{1}$ and $R_{2}$, which are kept at different temperatures $T_{1}$ and $T_{2}$, respectively. These temperatures are controlled by thermal baths, and $T_{2}$ is fixed at $296 \mathrm{~K}$, whereas $T_{1}$ can be set at a value between 88 and $296 \mathrm{~K}$ using the stratified vapor above a liquid nitrogen bath. In the figure, the two resistances have been drawn with their associated thermal noise generators $\eta_{1}$ and $\eta_{2}$, whose power spectral densities are given by the Nyquist formula $\left|\tilde{\eta}_{m}\right|^{2}=4 k_{B} R_{m} T_{m}$, with $m=1,2$ [see Eqs. (16) and (17)]. The coupling capacitance $C$ controls the electrical power exchanged between the resistances and, as a consequence, the energy exchanged between the two baths. No other coupling exists between the two resistances which are inside two separated screened boxes. The quantities $C_{1}$ and $C_{2}$ are the capacitances of the circuits and the cables. Two extremely low-noise amplifiers $A_{1}$ and $A_{2}$ [96] measure the voltage $V_{1}$ and $V_{2}$ across the resistances $R_{1}$ and $R_{2}$, respectively. All the relevant quantities considered in this paper can be derived by the measurements of $V_{1}$ and $V_{2}$, as we discuss below.

\section{Stochastic equations for the voltages}

We now proceed to derive the equations for the dynamical variables $V_{1}$ and $V_{2}$. Furthermore, we discuss how our system can be mapped onto a system with two interacting Brownian particles, in the overdamped regime, coupled to two different temperatures; see Fig. 8(b). Let $q_{m}(m=1,2)$ be the charges that have flowed through the resistances $R_{m}$, so that the instantaneous current flowing through them is $i_{m}=\dot{q}_{m}$. A circuit analysis shows that the equations for the charges are

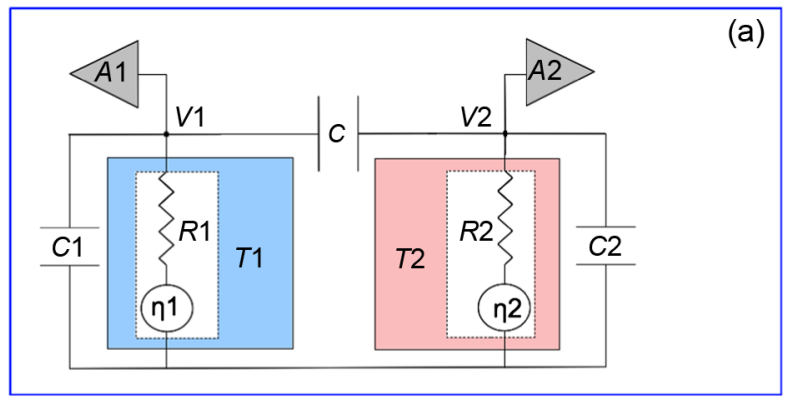

$$
\begin{aligned}
& R_{1} \dot{q}_{1}=-q_{1} \frac{C_{2}}{X}+\left(q_{2}-q_{1}\right) \frac{C}{X}+\eta_{1}, \\
& R_{2} \dot{q}_{2}=-q_{2} \frac{C_{1}}{X}+\left(q_{1}-q_{2}\right) \frac{C}{X}+\eta_{2},
\end{aligned}
$$

where $\eta_{m}$ is the usual white noise, $\left\langle\eta_{i}(t) \eta_{j}\left(t^{\prime}\right)\right\rangle=$ $2 \delta_{i j} k_{B} T_{i} R_{j} \delta\left(t-t^{\prime}\right)$, and where we have introduced the quantity $X=C_{2} C_{1}+C\left(C_{1}+C_{2}\right)$. Equations (16) and (17) are the same as those for the two coupled Brownian particles sketched in Fig. 8(b) when one regards $q_{m}$ as the displacement of the particle $m, i_{m}$ as its velocity, $K_{m}=$ $C_{m^{\prime}} / X\left(m^{\prime}=2\right.$ if $m=1$ and $m^{\prime}=1$ if $\left.m=2\right)$ as the stiffness of the spring $m, K=C / X$ as the coupling spring, and $R_{m}$ the viscosity term. The analogy with the Feymann ratchet can be made by assuming, as done in Ref. [82], that the particle $m_{1}$ has an asymmetric shape and on average moves faster in one direction than in the other one.

We now rearrange Eqs. (16) and (17) to obtain the Langevin equations for the voltages, which will be useful in the following discussion. The relationships between the measured voltages and the charges are

$$
\begin{aligned}
& q_{1}=\left(V_{1}-V_{2}\right) C+V_{1} C_{1}, \\
& q_{2}=\left(V_{1}-V_{2}\right) C-V_{2} C_{2} .
\end{aligned}
$$

By plugging Eqs. (18) and (19) into Eqs. (16) and (17), and rearranging terms, we obtain

$$
\begin{aligned}
& \left(C_{1}+C\right) \dot{V}_{1}=C \dot{V}_{2}+\frac{1}{R_{1}}\left(\eta_{1}-V_{1}\right), \\
& \left(C_{2}+C\right) \dot{V}_{2}=C \dot{V}_{1}+\frac{1}{R_{2}}\left(\eta_{2}-V_{2}\right) .
\end{aligned}
$$

FIG. 8. (a) Diagram of the circuit. The resistances $R_{1}$ and $R_{2}$ are kept at temperature $T_{1}$ and $T_{2}=296 \mathrm{~K}$, respectively. They are coupled via the capacitance $C$. The capacitances $C_{1}$ and $C_{2}$ schematize the capacitances of the cables and of the amplifier inputs. The voltages $V_{1}$ and $V_{2}$ are amplified by the two low-noise amplifiers $A_{1}$ and $A_{2}$ [96]. The other relevant parameters are $q_{m}(m=1,2)$, i.e., the charges that have flowed through the resistances $R_{m}$, and the instantaneous current flowing through them, i.e., $i_{m}=\left(d q_{m} / d t\right)$. (b) The circuit in (a) is equivalent to two Brownian particles $\left(m_{1}\right.$ and $\left.m_{2}\right)$ moving inside two different heat baths at $T_{1}$ and $T_{2}$. The two particles are trapped by two elastic potentials of stiffness $K_{1}$ and $K_{2}$ and coupled by a spring of stiffness $K$ [see text and Eqs. (16) and (17)]. The analogy is straightforward by considering $q_{m}$ the displacement of the particle $m, i_{m}$ its velocity, $K_{m}=C_{m^{\prime}} / X$ (with $m \neq m^{\prime}$ ) the stiffness of the spring $m$, and $K=C / X$ the coupling spring. 


\section{Thermodynamics quantities}

Two important quantities can be identified in the circuit depicted in Fig. 8: the energy $Q_{m, \tau}$ dissipated in each resistor in a time and the work $W_{m, \tau}$ exerted by one circuit on the other one in a time $\tau$. These two thermodynamic quantities are related to the internal energy variation in the time $\tau$ by the first principle:

$$
\Delta U_{m, \tau}=W_{m, \tau}-Q_{m, \tau} .
$$

Furthermore, it has been proved that the measured variance $\sigma_{m}^{2}$ of $V_{m}$ is related to the mean heat flux $\left\langle\dot{Q}_{m}\right\rangle \propto \partial_{t}\left\langle Q_{m}\right\rangle$ :

$$
\sigma_{m}^{2}=\left\langle V_{m}^{2}\right\rangle=\sigma_{m, \mathrm{eq}}^{2}+\left\langle\dot{Q}_{m}\right\rangle R_{m},
$$

where $\sigma_{m, \text { eq }}^{2}=k_{B} T_{m}\left(C+C_{m^{\prime}}\right) / X$ is the equilibrium value of $\sigma_{m}^{2}$. Note that $\left\langle\dot{Q}_{m}\right\rangle \propto\left(T_{m^{\prime}}-T_{m}\right)$; thus, in the equilibrium case $T_{m}=T_{m^{\prime}}$, and consequently $\left\langle\dot{Q}_{m}\right\rangle=0$.

We do not give here the exact expressions of $\Delta U_{m, \tau}$, $W_{m, \tau}, Q_{m, \tau}$, and $\sigma_{m}^{2}$, which have been computed and measured in Refs. [91,92]. We discuss instead how the FT is modified in the case of two heat baths.

\section{Fluctuation theorem for work and heat}

One expects that the thermodynamic quantities satisfy a fluctuation theorem of the type $[4,6,81,83,88-90]$

$$
\ln \frac{P\left(E_{m, \tau}\right)}{P\left(-E_{m, \tau}\right)}=\beta_{12} E_{m, \tau} \Sigma(\tau),
$$

where $E_{m, \tau}$ stands for either $W_{m, \tau}$ or $Q_{m, \tau}, \beta_{12}=$ $\left(1 / T_{1}-1 / T_{2}\right) / k_{B}$, and $\Sigma(\tau) \rightarrow 1$ for $\tau \rightarrow \infty$. Equation (24) has been proven in Ref. [92].

As the system is in a stationary state, we have $\left\langle W_{m, \tau}\right\rangle=\left\langle Q_{\tau, m}\right\rangle$. On the contrary, the comparison of the PDF of $W_{m, \tau}$ with those of $Q_{\tau, m}$, measured at various temperatures, presents several interesting features. In Fig. 9(a), we plot $P\left(W_{1, \tau}\right), P\left(-W_{2, \tau}\right), P\left(Q_{1, \tau}\right)$, and $P\left(-Q_{2, \tau}\right)$ measured in equilibrium at $T_{1}=T_{2}=296 \mathrm{~K}$ and $\tau \simeq 0.1 \mathrm{~s}$. We immediately see that the fluctuations of the work are almost Gaussian, whereas those of the heat present large exponential tails. This well-known difference [48] between $P\left(Q_{m, \tau}\right)$ and $P\left(W_{m, \tau}\right)$ is induced by the fact that $Q_{m, \tau}$ depends also on $\Delta U_{m, \tau}$ [Eq. (22)], which is the sum of the square of Gaussian distributed variables, thus inducing exponential tails in $P\left(Q_{m, \tau}\right)$. In Fig. 9(a), we also notice that $P\left(W_{1, \tau}\right)=P\left(-W_{2, \tau}\right)$ and $P\left(Q_{1, \tau}\right)=P\left(-Q_{2, \tau}\right)$, showing that in equilibrium all fluctuations are perfectly symmetric. The same PDFs measured in the out-of-equilibrium case at $T_{1}=88 \mathrm{~K}$ are plotted in Fig. 9(b). We notice here that in this case the behavior of the PDFs of the heat is different from those of the work. Indeed, although $\left\langle W_{m, \tau}\right\rangle>0$, we observe that $P\left(W_{1, \tau}\right)=P\left(-W_{2, \tau}\right)$, while $P\left(Q_{1, \tau}\right) \neq P\left(-Q_{2, \tau}\right)$. Indeed, the shape of $P\left(Q_{1, \tau}\right)$ is strongly modified by changing $T_{1}$ from 296 to $88 \mathrm{~K}$; whereas the shape of $P\left(-Q_{2, \tau}\right)$ is slightly modified by the large temperature change, only the tails of $P\left(-Q_{2, \tau}\right)$ present a small asymmetry testifying to the presence of a small heat flux. The fact that $P\left(Q_{1, \tau}\right) \neq P\left(-Q_{2, \tau}\right)$, whereas $P\left(W_{1, \tau}\right)=P\left(-W_{2, \tau}\right)$, can be understood by noticing that $Q_{m, \tau}=W_{m, \tau}-\Delta U_{m, \tau}$. Indeed, $\Delta U_{m, \tau}$ [Eq. (22)] depends on the values of $C_{m}$ and $V_{m}^{2}$. As $C_{1} \neq$ $C_{2}$ and $\sigma_{2} \geq \sigma_{1}$, this explains the different behavior of $Q_{1}$ and $Q_{2}$. On the contrary, $W_{m}$ depends only on $C$ and the product $V_{1} V_{2}$.

We study whether our data satisfy the fluctuation theorem as given by Eq. (24) in the limit of large $\tau$. It turns out that the symmetry imposed by Eq. (24) is reached for rather small $\tau$ for $W$. On the contrary, it converges very slowly for $Q$. We have only a qualitative argument to explain this difference in the asymptotic behavior: by looking at the data one understands that the slow convergence is induced by the presence of the exponential tails of $P\left(Q_{1, \tau}\right)$ for small $\tau$.

To check Eq. (24), we plot in Fig. 9(c) the symmetry function $\operatorname{Sym}\left(E_{1, \tau}\right)=\ln \left[P\left(E_{1, \tau}\right) / P\left(-E_{1, \tau}\right)\right]$ as a function of $E_{1, \tau} /\left(k_{B} T_{2}\right)$ measured at different $T_{1}$, but $\tau=0.1 \mathrm{~s}$ for $\operatorname{Sym}\left(W_{1, \tau}\right)$ and $\tau=2 s$ for $\operatorname{Sym}\left(Q_{1, \tau}\right)$. Indeed, $\operatorname{Sym}\left(Q_{1, \tau}\right)$ reaches the asymptotic regime only for $\tau \rightarrow 2 s$. We see that $\operatorname{Sym}\left(W_{1, \tau}\right)$ is a linear function of $W_{1, \tau} /\left(k_{B} T_{2}\right)$ at all $T_{1}$. These straight lines have a slope $\alpha\left(T_{1}\right)$ which, according to Eq. (24), should be $\left(\beta_{12} k_{B} T_{2}\right)$. In order to check this prediction we fit the slopes of the straight lines in Fig. 9(c). From the fitted $\alpha\left(T_{1}\right)$ we deduce a temperature $T_{\text {fit }}=T_{2} /\left[\alpha\left(T_{1}\right)+1\right]$, which is compared to the measured temperature $T_{1}$ in Fig. 9(d). In this figure, the straight line of slope 1 indicates that $T_{\text {fit }} \simeq T_{1}$ within a few percent. These experimental results indicate that our data verify the fluctuation theorem, Eq. (24), for the work and the heat, but that the asymptotic regime is reached for much larger time for the latter.

\section{Entropy production rate}

It is now important to analyze the entropy produced by the total system, circuit plus heat reservoirs. We consider first the entropy $\Delta S_{r, \tau}$ due to the heat exchanged with the reservoirs, which reads $\Delta S_{r, \tau}=Q_{1, \tau} / T_{1}+Q_{2, \tau} / T_{2}$. This entropy is a fluctuating quantity as both $Q_{1}$ and $Q_{2}$ fluctuate, and its average in a time $\tau$ is $\left\langle\Delta S_{r, \tau}\right\rangle=$ $\left\langle Q_{r, \tau}\right\rangle\left(1 / T_{1}-1 / T_{2}\right)=A \tau\left(T_{2}-T_{1}\right)^{2} /\left(T_{2} T_{1}\right)$. However, the reservoir entropy $\Delta S_{r, \tau}$ is not the only component of the total entropy production: one has to take into account the entropy variation of the system, due to its dynamical evolution. Indeed, the state variables $V_{m}$ also fluctuate as an effect of the thermal noise, and thus, if one measures their values at regular time interval, one obtains a "trajectory" in the phase space $\left[V_{1}(t), V_{2}(t)\right]$. Thus, following Seifert [11], 

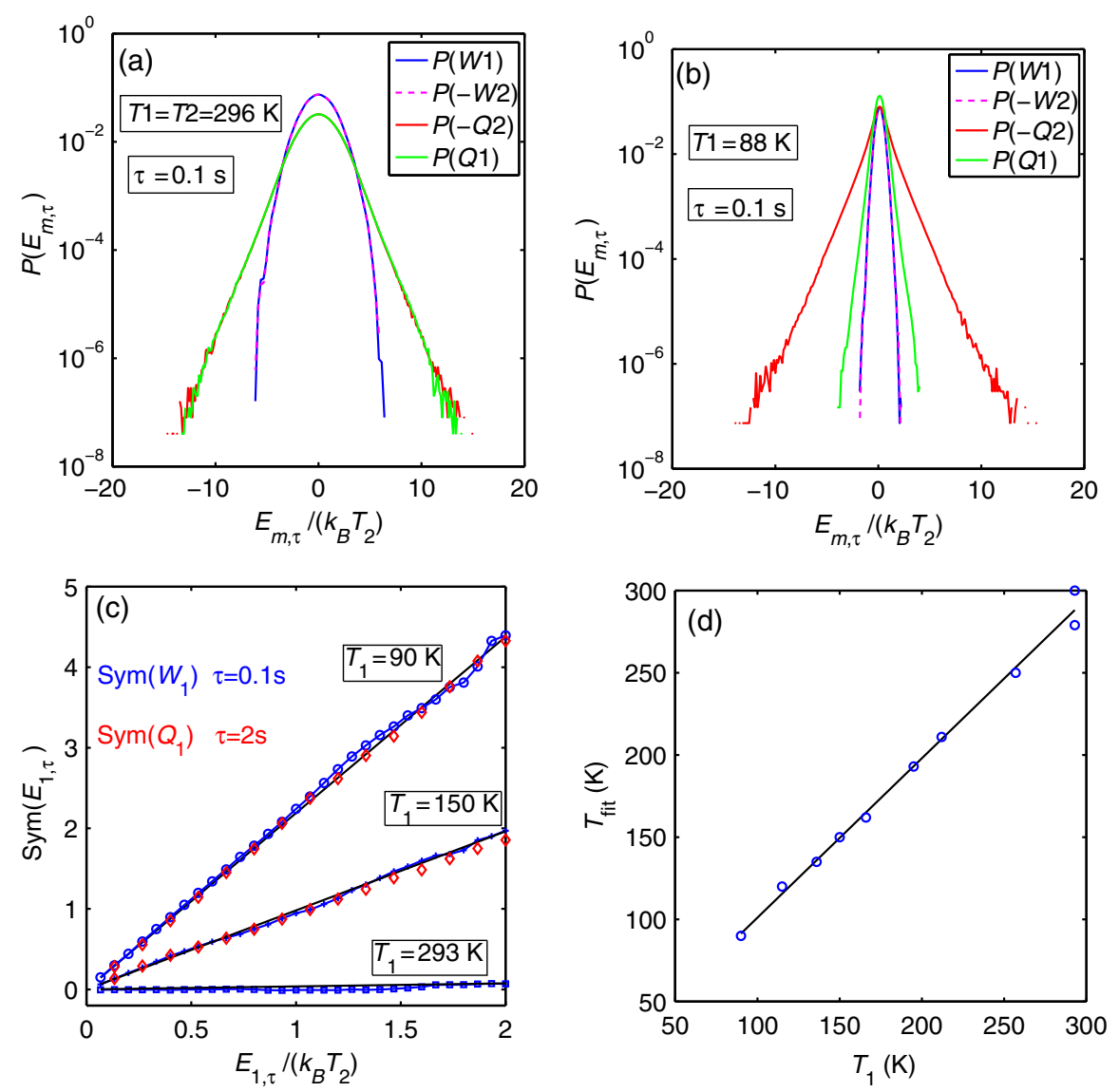

FIG. 9. (a) Equilibrium: $P\left(W_{m, \tau}\right)$ and $P\left(Q_{m, \tau}\right)$, measured in equilibrium at $T_{1}=T_{2}=296 \mathrm{~K}$ and $\tau=0.1 s$, are plotted as functions of $E$, where $E$ stands for either $W$ or $Q$. Notice that when the system is in equilibrium, $P\left(W_{1, \tau}\right)=P\left(-W_{2, \tau}\right)$ and $P\left(Q_{1, \tau}\right)=P\left(-Q_{2, \tau}\right)$. (b) Out of equilibrium: Same distributions as in (a) but the PDFs are measured at $T_{1}=88 \mathrm{~K}, T_{2}=296 \mathrm{~K}$, and $\tau=0.1 \mathrm{~s}$. Notice that when the system is out of equilibrium, $P\left(W_{1, \tau}\right)=P\left(-W_{2, \tau}\right)$ but $P\left(Q_{1, \tau}\right) \neq P\left(-Q_{2, \tau}\right)$. The reason for this difference is explained in the text. (c) The symmetry function $\operatorname{Sym}\left(E_{1, \tau}\right)$, measured at various $T_{1}$, is plotted as a function of $E_{1}\left(W_{1}\right.$ or $\left.Q_{1}\right)$. The theoretical slope of these straight lines is $T_{2} / T_{1}-1$. (d) The temperature $T_{\text {fit }}$ estimated from the slopes of the lines in (c) is plotted as a function of the $T_{1}$ measured by the thermometer. The slope of the line is 1 , showing that $T_{\text {fit }} \simeq T_{1}$ within a few percent.

who developed this concept for a single heat bath, one can introduce a trajectory entropy for the evolving system $S_{s}(t)=-k_{B} \log P\left[V_{1}(t), V_{2}(t)\right]$, which extends to nonequilibrium systems the standard Gibbs entropy concept. Therefore, when evaluating the total entropy production, one has to take into account the contribution over the time interval $\tau$ of

$$
\Delta S_{s, \tau}=-k_{B} \log \left[\frac{P\left[V_{1}(t+\tau), V_{2}(t+\tau)\right]}{P\left[V_{1}(t), V_{2}(t)\right]}\right]
$$

It is worth noting that the system we consider is in a nonequilibrium steady state, with a constant external driving $\Delta T$. Therefore, the probability distribution $P\left(V_{1}, V_{2}\right)$ does not depend explicitly on the time, and $\Delta S_{s, \tau}$ is nonvanishing whenever the final point of the trajectory is different from the initial one: $\left[V_{1}(t+\tau)\right.$, $\left.V_{2}(t+\tau)\right] \neq\left[V_{1}(t), V_{2}(t)\right]$. Thus, the total entropy change reads $\Delta S_{\text {tot }, \tau}=\Delta S_{r, \tau}+\Delta S_{s, \tau}$, where we omit the explicit dependence on $t$, as the system is in a steady state, as discussed above. This entropy has several interesting features. The first one is that $\left\langle\Delta S_{s, \tau}\right\rangle=0$, and as a consequence, $\left\langle\Delta S_{\text {tot }}\right\rangle=\left\langle\Delta S_{r}\right\rangle$, which grows with increasing $\Delta T$. The second and most interesting result is that independently of $\Delta T$ and of $\tau$, the following equality always holds:

$$
\left\langle\exp \left(-\Delta S_{\text {tot }} / k_{B}\right)\right\rangle=1,
$$

for which we find experimental evidence, as discussed in the following, and provide a theoretical proof in Refs. [91,92]. Equation (26) represents an extension to two temperature sources of the result obtained for a system in a single heat bath driven out of equilibrium by a timedependent mechanical force [5,11], and our results provide the first experimental verification of the expression in a system driven by a temperature difference. Equation (26) implies that $\left\langle\Delta S_{\text {tot }}\right\rangle \geq 0$, as prescribed by the second law. From symmetry considerations, it follows immediately 

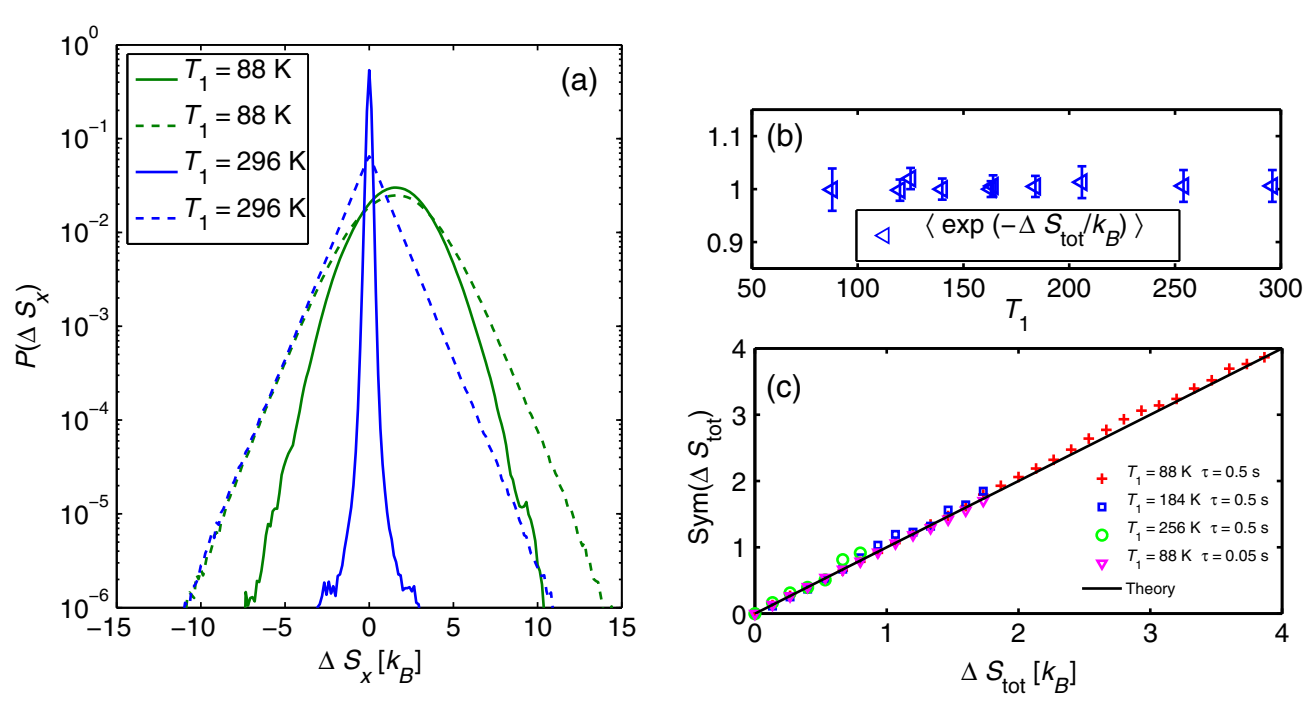

FIG. 10. (a) The probability $P\left(\Delta S_{r}\right)$ (dashed lines) and $P\left(\Delta S_{\text {tot }}\right)$ (continuous lines) measured at $T_{1}=296 \mathrm{~K}$ (blue line) which corresponds to equilibrium and $T_{1}=88 \mathrm{~K}$ (green lines) out of equilibrium. Notice that both distributions are centered at zero at equilibrium and shifted towards positive value in the out of equilibrium. (b) $\left\langle\exp \left(-\Delta S_{\text {tot }}\right)\right\rangle$ as a function of $T_{1}$ at two different $\tau=0.5 s$ and $\tau=0.1 s$. (c) Symmetry function $\operatorname{Sym}\left(\Delta S_{\text {tot }}\right)=\log \left[P\left(\Delta S_{\text {tot }}\right) / P\left(-\Delta S_{\text {tot }}\right)\right]$ as a function of $\Delta S_{\text {tot }}$. The black straight line of slope 1 corresponds to the theoretical prediction.

that, at equilibrium $\left(T_{1}=T_{2}\right)$, the probability distribution of $\Delta S_{\text {tot }}$ is symmetric: $P_{\text {eq }}\left(\Delta S_{\text {tot }}\right)=P_{\text {eq }}\left(-\Delta S_{\text {tot }}\right)$. Thus, Eq. (26) implies that the probability density function of $\Delta S_{\text {tot }}$ is a Dirac $\delta$ function when $T_{1}=T_{2}$; i.e., the quantity $\Delta S_{\text {tot }}$ is rigorously zero in equilibrium, both in average and fluctuations, and so its mean value and variance provide a measure of the entropy production. The measured probabilities $P\left(\Delta S_{r}\right)$ and $P\left(\Delta S_{\text {tot }}\right)$ are shown in Fig. 10(a). We see that $P\left(\Delta S_{r}\right)$ and $P\left(\Delta S_{\text {tot }}\right)$ are quite different and that the latter is close to a Gaussian and reduces to a Dirac $\delta$ function in equilibrium, i.e., $T_{1}=T_{2}=296 \mathrm{~K}$ [notice that, in Fig. 10(a), the small broadening of the equilibrium $P\left(\Delta S_{\text {tot }}\right)$ is just due to unavoidable experimental noise and discretization of the experimental probability density functions]. The experimental measurements satisfy Eq. (26) as it is shown in Fig. 10(b). It is worth noting that Eq. (26) implies that $P\left(\Delta S_{\text {tot }}\right)$ should satisfy a fluctuation theorem of the form $\log \left[P\left(\Delta S_{\text {tot }}\right) / P\left(-\Delta S_{\text {tot }}\right)\right]=\Delta S_{\text {tot }} / k_{B}, \forall \tau, \Delta T$, as discussed extensively in Refs. [2,53]. We clearly see in Fig. 10(c)that this relation holds for different values of the temperature gradient. Thus, this experiment clearly establishes a relationship between the mean and the variance of the entropy production rate in a system driven out of equilibrium by the temperature difference between two thermal baths coupled by electrical noise. Because of the formal analogy with Brownian motion, the results also apply to mechanical coupling $[95,97,98]$.

\section{B. Entropy production in a single-electron box}

Another interesting experiment on the measure of the entropy production in a system subjected to a temperature difference is presented in Ref. [93]. We summarize here the main results. The experimental system is sketched in Fig. 11, and it is based on a single-electron box at low temperature. This is an excellent test benchmark for thermodynamics in small systems $[99,100]$, and an interesting review of the statistical properties of coupled circuits, both quantum and classical, can be found in Ref. [101].

In the single-electron box shown in Fig. 11(a) the electrons in the normal metal copper island $(N)$ can tunnel to the superconducting $\mathrm{Al}$ island $(S)$ through the aluminium oxide insulator $(I)$. The integer net number of electrons tunneled from $S$ to $N$ is denoted by $n$. This number, monitored by the nearby single-electron transistor (SET) shown in Fig. 11(a), is the classical system degree of freedom.

Indeed, the device in Fig. 11(a) can be represented with a classical electric circuit, in which the energy stored in the capacitors and in the voltage sources can be exactly measured [99]. As in the previous section, Sec. VA 1, the conductor $N$ and $S$ are not at the same temperature. Furthermore, here the system is driven by a voltage $V_{g}$ which oscillates much slower than the relaxation time of the device. Thus, the forward and backward processes from the maximum to the minimum of $V_{g}$ can be considered. By the measured values of $n(t)$ and $V_{g}$, one can estimate the heats, $Q_{N}$ and $Q_{S}$, exchanged by the two heat baths in a time $t_{f}$, which is the period of the driving signal. In this way the thermal entropy $\Delta S^{\text {th }}=Q_{N} / T_{N}+Q_{S} / T_{S}$ can be computed. Furthermore, the trajectory-dependent entropy can be estimated by measuring $\Delta s=-k_{b} \log \left[P\left(n\left(t_{f}\right)\right) /\right.$ $P(n(0))$ ], where $P(n(t))$ is the probability that at time $t$ the system is in the state $n(t)$ for a value of the driving $V_{g}(t)$. 
(a)

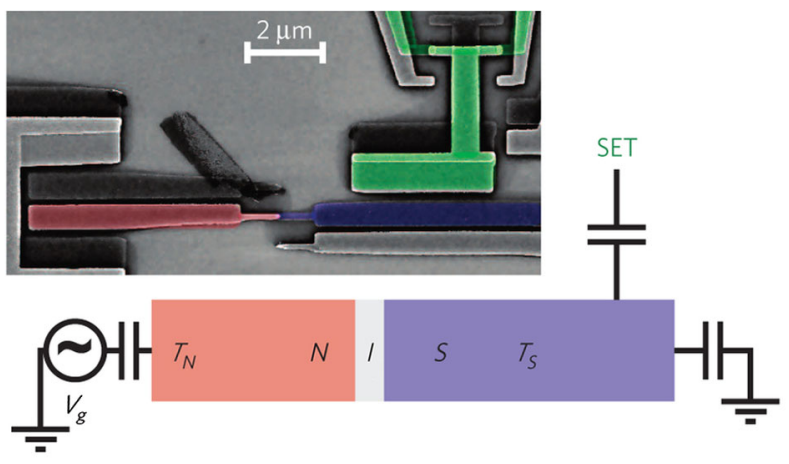

(b)

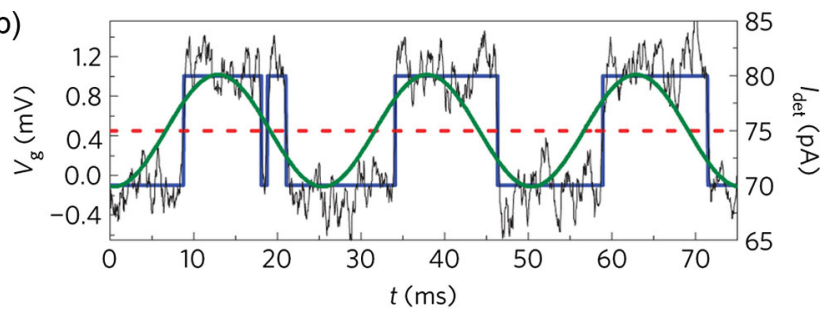

FIG. 11. (a) Sketch of the measured system together with a scanning electron micrograph of a typical sample. The colors on the micrograph indicate the correspondingly colored circuit elements in the sketch. (b) Typical trace of the measured detector signal under a sinusoidal protocol for the drive $V_{g}$, plotted in green. This trace covers three realizations of the forward protocol ( $V_{g}$ from -0.1 to $1 \mathrm{mV}$ ), and three realizations of the backward protocol $\left(V_{g}\right.$ from 1 to $\left.-0.1 \mathrm{mV}\right)$. The SET current $I_{\text {det }}$, plotted in black, indicates the charge state of the box. The output of the threshold detection is shown in solid blue, with the threshold level indicated by the dashed red line (adapted from Ref. [93]).
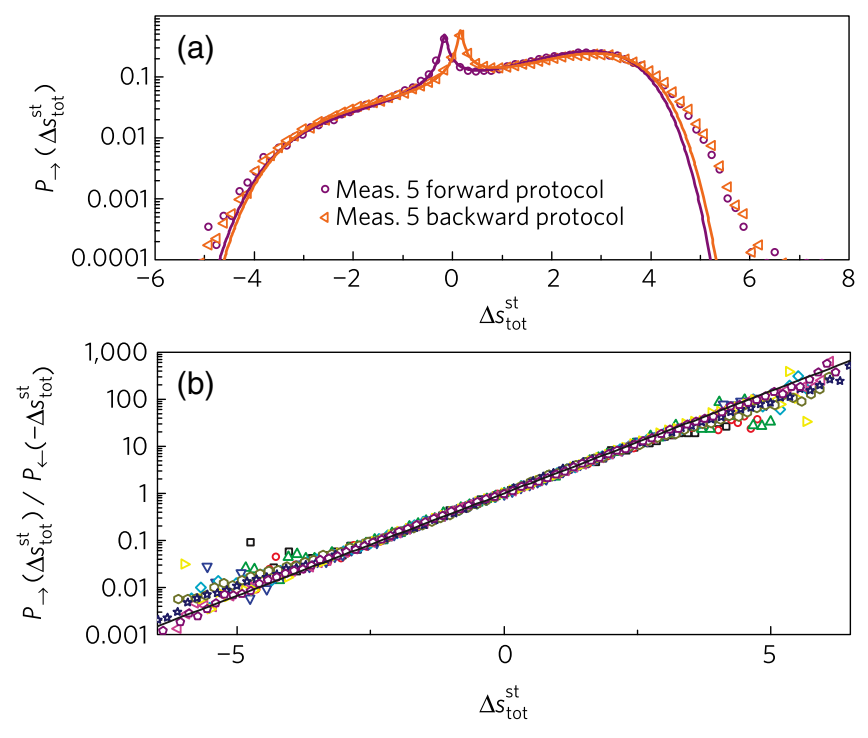

FIG. 12. (a) Probability distribution of the total entropy $\Delta S_{\text {tot }}$, which has been measured in the circuit shown in Fig. 11 and described in the text. (b) The symmetry functions $\operatorname{Sym}\left(\Delta S_{\text {tot }}\right)=$ $\log \left[P\left(\Delta S_{\text {tot }}\right) / P\left(-\Delta S_{\text {tot }}\right)\right]$ of $P\left(\Delta S_{\text {tot }}\right)$ as a function of $\Delta S_{\text {tot }}$. In spite of the highly non-Gaussian nature of $P\left(\Delta S_{\text {tot }}\right)$, we see that $\operatorname{Sym}\left(\Delta S_{\text {tot }}\right)=k_{B} \Delta S_{\text {tot }}$ (adapted from Ref. [93]).
The total entropy is, of course, $\Delta S_{\text {tot }}=\Delta S^{\text {th }}+\Delta S$, and its probability distribution $P\left(\Delta S_{\text {tot }}\right)$ can be measured. The results for the forward and back processes are shown in Fig. 12(a), and the corresponding symmetry functions $\operatorname{Sym}\left(\Delta S_{\text {tot }}\right)=\log \left[P\left(\Delta S_{\text {tot }}\right) / P\left(-\Delta S_{\text {tot }}\right)\right]$ are plotted Fig. 12(b). In spite of the fact that $P\left(\Delta S_{\text {tot }}\right)$ are highly nonGaussian, we notice that $\operatorname{Sym}\left(\Delta S_{\text {tot }}\right)=k_{B} \Delta S_{\text {tot }}$, which implies that Eq. (26) is also satisfied by these data.

As in the previous section, Sec. VA 5, the main result of this experiment is that stochastic entropy production extracted from the trajectories is related to thermodynamic entropy production from dissipated heat in the respective thermal baths.

\section{MOTOR POWER AND EFFICIENCY}

Historically, one of the main purposes of thermodynamics has been the study of the efficiency of thermal machines and power plants. Nowadays there is a wide interest in extending these studies to micro and nano motors which play a major role in biological mechanisms and small devices. In Sec. II B, we see that in small systems all of the thermodynamics quantities fluctuate. Thus, we are interested in knowing the influence of these fluctuations on the efficiency of small devices where the dissipated energies and the produced work are a few $k_{B} T$. Furthermore, it is useful to know this efficiency at the maximum power and not in the quasistatic regimes, such as the Carnot cycle, where the produced power is close to zero. These important questions have been theoretically studied in several articles $[2,102-107]$ and only in a few proof-of-principle experiments [108-111]. The first stochastic Carnot machine was reported in Ref. [108]. In this experiment a Brownian particle trapped by an optical tweezer is subjected to a kind of Carnot cycle, inspired by a theoretical model proposed in Ref. [103]. The cycle, used in a very similar experiment [109], is sketched in Fig. 13(a), which we describe in some detail. The Brownian particle is trapped by a harmonic potential [bottom row in Fig. 13(a)] whose stiffness is changed as a function of time. The increase of the stiffness is equivalent to a compression (the motion of the particle is more confined), the decrease to an expansion. In the experiment the bead is subjected to a random force which plays the role of an effective temperature, which can be easily changed by changing the amplitude of the random forcing. As in the Carnot cycle, the cycle in Fig. 13(a) is composed by an isothermal and an adiabatic compression and by an isothermal and an adiabatic expansion. Notice that the construction of adiabatic processes for a Brownian particle is a real challenge, which has been achieved by changing simultaneously the temperature in such a way that the exchanged heat, during the adiabatics, is zero on average (see Refs. $[109,112]$ for details). The work and the heat in this experiment are computed as described in Secs. II B, IV, and V. As these two quantities fluctuate, the contribution of the fluctuations during adiabatics must 
(a)

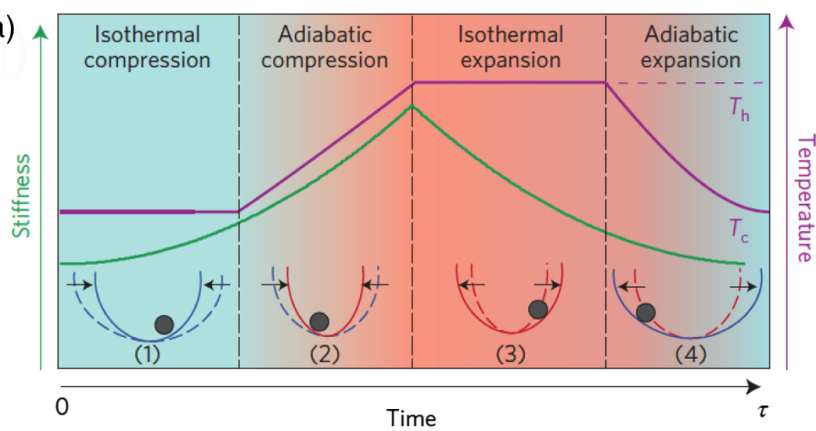

(b)

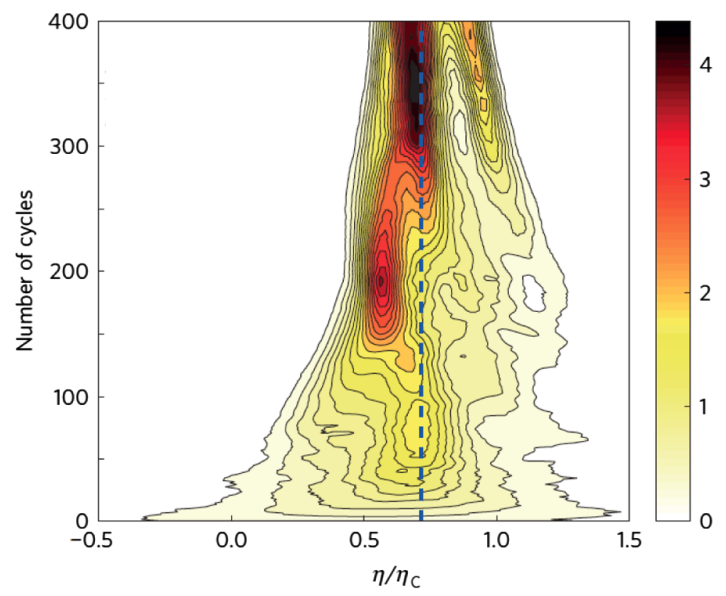

FIG. 13. (a) Schematic of the Carnot cycle applied to a Brownian particle trapped in a harmonic potential by a laser beam. The bottom row indicates the harmonic potential as a function of time. The stiffness of the potential is controlled by the laser intensity, as indicated by the green line. A random force, applied to the particle, plays the role of an effective temperature whose value is changed as indicated by the magenta line. (b) Contour lines of the probability distribution of the cycle efficiency averaged on $n$ number of cycles. The efficiency $\eta$ is normalized to the Carnot efficiency $\eta_{c}$. The black dashed line indicates the mean value of $\eta / \eta_{c}$ (adapted from Ref. [109]).

be taken into account in computing the efficiency, which is defined as $\eta_{\text {cycle }}=W_{\text {cycle }} / Q_{\text {hot }}$, where $W_{\text {cycle }}$ is the work produced during a cycle and $Q_{\text {hot }}$ is the heat absorbed from the hot sources. Because of $W$ and $Q$ fluctuations, $\eta_{\text {cycle }}$ also fluctuates a lot because, as we have seen in previous sections, $Q$ can be zero and even negative. The measured probability distribution of $\eta_{\text {cycle }}$ is plotted in Fig. 13(b) as function of the number of cycles used to average it. The efficiency $\eta_{\text {cycle }}$ is normalized to the standard Carnot efficiency, $\eta_{c}=1-T_{\text {cold }} / T_{\text {hot }}$. Although the mean value $\left\langle\eta_{\text {cycle }}\right\rangle$ is smaller than $\eta_{c}$, we clearly see that, for a small number of cycles, $\eta$ has big fluctuations which extend from values much larger than $\eta_{c}$ to negative values. Furthermore, as the cycle is performed in finite time, the power produced by the system can be computed as a function of the cycle duration. This power has a maximum, and the other interesting result of this experiment is that the mean efficiency at the maximum power follows the CurzonAhlborn expression $\left\langle\eta_{\text {cycle }}\right\rangle=1-\sqrt{T_{\text {cold }} / T_{\text {hot }}}[102]$.
Another interesting article [113] presents the theoretical and experimental results on the conversion of one form of work to another. Using a Brownian particle as an isothermal machine driven by two independent periodic forces, the authors of Ref. [113] analytically compute and experimentally measure the stochastic thermodynamic properties of this Brownian engine. Specifically, the efficiency of the energy transfer between the two driving forces and the Onsager coefficients of the coupling are evaluated.

The results of these experiments show the kind of problems that one encounters in the study of the efficiency of nano devices. For example, the study of the efficiency at maximum power and the behavior of $\eta$ fluctuations have been subjects of extensive theoretical investigation $[2,104-106]$, to understand their system dependence and eventually their universality. We do not discuss these theoretical results because they are far from the purposes of this review. The interested reader can look at the abovementioned references. However, it is worth mentioning that several small devices, such as a molecular motor, are driven by chemical reaction, and the efficiency of these devices has been studied theoretically [107,114-116], but to my knowledge no proof-of-principle experiment, as the one presented here, has been performed for this chemically driven system.

\section{FLUCTUATION DISSIPATION RELATIONS FOR NESS}

As we see in the previous section, current theoretical developments in nonequilibrium statistical mechanics have led to significant progress in the study of systems around states far from thermal equilibrium. Systems in nonequilibrium steady states are the simplest examples because the dynamics of their degrees of freedom $x$ under fixed control parameters $\lambda$ can be statistically described by time-independent probability densities $\rho_{0}(x, \lambda)$. NESSs naturally occur in mesoscopic systems such as colloidal particles dragged by optical tweezeres, Brownian ratches, and molecular motors because of the presence of nonconservative or time-dependent forces [117]. At these length scales fluctuations are important, so it is essential to establish a quantitative link between the statistical properties of the NESS fluctuations and the response of the system to external perturbations. Around thermal equilibrium this link is provided by the fluctuation-dissipation theorem [34].

The validity of the FDR in systems out of thermal equilibrium has been the subject of intensive study during recent years. We recall that for a system in equilibrium with a thermal bath at temperature $T$ the FDR establishes a simple relation between the two-time correlation function $C(t-s)$ of a given observable and the linear response function $R(t, s)$ of this observable to a weak external perturbation, 


$$
\partial_{s} C(t, s)=k_{B} T R(t, s),
$$

where in equilibrium $C(t, s)$ and $R(t, s)$ depend only on the time difference $(t-s)$. However, Eq. (27) is not necessarily fulfilled out of equilibrium and violations are observed in a variety of systems, such as glassy materials [24,118-123], granular matter [124], biological systems [125], and resonators [126].

This motivated a theoretical and experimental work devoted to a search of a general framework describing FD relations; see the review Ref. [34]. The generalization of the fluctuation-dissipation theorem around NESS for systems with Markovian dynamics has been achieved from different theoretical approaches [17,19,20,127-136]. The different generalized formulations of FDR link correlation functions of the fluctuations of the observable of interest $O(x)$ in the unperturbed NESS with the linear response function of $O(x)$ due to a small external time-dependent perturbation around the NESS. The observables involved in such relations are not unique, but they are equivalent in the sense that they lead to the same values of the linear response function. These theoretical relations may be useful in experiments and simulations to know the linear response of the system around NESS. Indeed, the response can be obtained from measurements entirely done at the unperturbed NESS of the system of interest without any need to perform the actual perturbation. Nevertheless, the theoretical equivalence of the different observables involved in those relations does not translate into equivalent experimental accessibility: e.g., strongly fluctuating observables such as instantaneous velocities may lead to large statistical errors in the measurements [137]. Additionally, NESS quantities themselves, such as local mean velocities, joint stationary densities, and the stochastic entropy, are not in general as easily measurable as dynamical observables directly related to the degrees of freedom [18]. Hence, before implementing the different fluctuation-response formulas in real situations, it is important to test its experimental validity under very well controlled conditions and to assess the influence of finite data analysis. The experimental test of some fluctuation-dissipation relations has been recently done in Refs. [18,61,137,138] for colloidal particles in toroidal optical traps and in systems subjected to thermal gradients $[139,140]$. We do not describe here specific experimental results which have already been widely discussed in the abovementioned articles (see also Refs. [2,3,34]). What is important to recall is that this term is related to the out-ofequilibrium current of the system, which is proportional to the mean total entropy production for a NESS.

\section{THERMODYNAMICS, INFORMATION, AND THE MAXWELL DEMONS}

The relationship between stochastic thermodynamics and information now has an increasing importance both theoretically and experimentally. This relationship is related to the famous paradox of a Maxwell's demon, which is an intelligent creature able to monitor individual molecules of a gas contained in two neighboring chambers $[141,142]$. Initially, the two chambers are at the same temperature, defined by the mean kinetic energy of the molecules and proportional to their mean-square velocity. Some of the particles, however, travel faster than others. By opening and closing a molecule-sized trapdoor in the partitioning wall, the demon can collect the faster molecules in one chamber and the slower ones in the other. The two chambers then contain gases with different temperatures, and that temperature difference may be used to power a heat engine and produce mechanical work. By gathering information about the particles positions and velocities and using that knowledge to sort them, the demon is able to decrease the entropy of the system and convert information into energy. Assuming the trapdoor is frictionless, the demon is able to do all that without performing any work himself in an apparent violation of the second law of thermodynamics. This paradox has originated a long debate on the connection between information and thermodynamics. A solution of the problem was proposed in 1929 by Leo Szilard, who used a simplified one-particle engine to explain it. This gedanken experiment can nowadays be realized [141].

\section{A. Szilard engine: Work production from information}

Modern technologies allow us to realize these gedanken experiments related to the Maxwell's demon original idea.

\section{Sizlard engine}

For example, a Szilard engine was realized in 2010 [143] by using a single microscopic Brownian particle in a fluid and confined to a spiral-staircase-like potential shown in Fig. 14. Driven by thermal fluctuations, the particle performs an erratic up and down motion along the staircase. However, because of the potential gradient, downward steps will be more frequent than upward steps, and the particle will on average fall down. The position of the particle is measured with the help of a CCD camera. Each time the particle is observed to jump upwards, this information is used to insert a potential barrier that hinders the particle to move down. By repeating this procedure, the average particle motion is now upstairs and work is done against the potential gradient. By lifting the particle, mechanical work has therefore been produced by gathering information about its position. This is the first example of a device that converts information into energy for a system coupled to a single thermal environment. However, there is not a contradiction with the second law because Sagawa and Ueda [144] formalized the idea that information gained through microlevel measurements can be used to extract added work from a heat engine. Their formula for the maximum extractable work is 
(a)

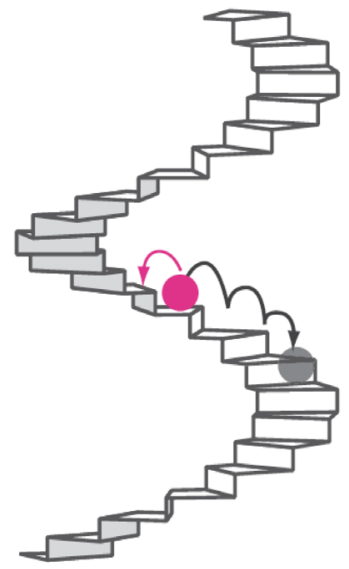

(b)

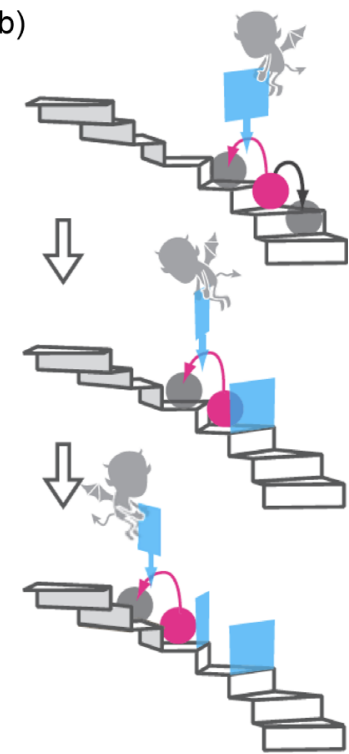

FIG. 14. (a) Experimental realization of Szilard's engine. (a) A colloidal particle in a staircase potential moves downward on average, but energy fluctuations can push it upward from time to time. (b) When the demon observes such an event, it inserts a wall to prevent downward steps. By repeating this procedure, the particle can be brought to move upwards, performing work against the force created by the staircase potential. In the actual experiment, the staircase potential is implemented by a tilted periodic potential and the insertion of the wall is simply realized by switching the potential, replacing a minimum (no wall) by a maximum (wall) (adapted from Ref. [143]).

$$
W_{\max }=-\Delta F+k_{B} T\langle I\rangle,
$$

where $\Delta F$ is the free-energy difference between the final and initial state and the extra term represents the so-called mutual information $I$. In the absence of measurement errors this quantity reduces to the Shannon entropy: $I=-\sum_{k} P\left(\Gamma_{k}\right) \ln \left[P\left(\Gamma_{k}\right)\right]$, where $P\left(\Gamma_{m}\right)$ is the probability of finding the system in the state $\Gamma_{k}$. Then in the specific case of the previously described staircase potential [143]: $I=-p \ln p-(1-p) \ln p$, where $p$ is the probability of finding the particle in a specific region.

In this context the Jarzynski equality discussed in Sec. IV also contains this extra term and it becomes

$$
\langle\exp (-\beta W+I)\rangle=\exp (-\beta \Delta F),
$$

which leads to

$$
\langle W\rangle \geq \Delta F-k_{B} T\langle I\rangle .
$$

Equations (29) and (30) generalize the second law of thermodynamics, taking into account the amount of information introduced into the system [142,145]. Indeed Eq. (30) indicates that, thanks to information, the work performed on the system to drive it between an initial and a

final equilibrium state can be smaller than the free-energy difference between the two states.

Equation (29) has been directly tested in a singleelectron transistor [146], similar to the one described in Sec. VIII A.

\section{Autonomous Maxwell's demon improves cooling}

In the previous section, Sec. VIII A, the Maxwell's demon has been realized using an external feedback. However, working at low temperature and coupling in a suitable way the single-electron devices, already described in Sec. VIII A, one can construct a local feedback which behaves as an autonomous Maxwell's demon and allows an efficient cooling of the system $[147,148]$. The device, whose principle is sketched in Fig. 15(a), is composed by a SET formed by a small normal metallic island connected to two normal metallic leads by tunnel junctions, which permit electron transport between the leads and the island. The SET is biased by a potential $V$ and a gate voltage $V_{g}$, applied to the island via a capacitance, controls the current $I$ flowing through the SET. The island is coupled capacitively with a singleelectron box which acts as a demon which detects the presence of an electron in the island and applies a feedback. Specifically, when an electron tunnels to the island, the demon traps it with a positive charge [illustrations 1 and 2 in Fig. 15(a)]. Conversely, when an electron leaves the island, the demon applies a negative charge to repel further electrons that would enter the island [illustrations 3 and 4 in Fig. 15(a)]. This effect is obtained by designing the electrodes of the demon in such a way that when an electron enters the island from a source electrode, an electron tunnels out of the demon island as a response, exploiting the mutual Coulomb repulsion between the two electrons. Similarly, when an electron enters to the drain electrode from the system island, an electron tunnels back to the demon island, attracted by the overall positive charge. The cycle of these interactions between the two devices realizes the autonomous demon, which allows the cooling of the leads. In the experimental realization presented in Ref. [147], the leads and the demon were thermally insulated, and the measurements of their temperatures is used to characterize the effect of the demon on the device operation. In Fig. 15(b) we plot the variation of the lead temperatures as a function of $n_{g} \propto V_{g}$ when the demon acts on the system. We clearly see that around $n_{g}=1 / 2$ the two leads are both cooled of $1 \mathrm{mK}$ at a mean temperature of $50 \mathrm{mK}$. This occurs because the tunneling electrons have to take the energy from the thermal energy of the leads, which, being thermally isolated, cool down. This increases the rate at which electrons tunnel against Coulomb repulsion, giving rise to increased cooling power. At the same time, the demon increases its temperature because it has to dissipate energy in order to process information, as discussed in Ref. [149]. 
(a)

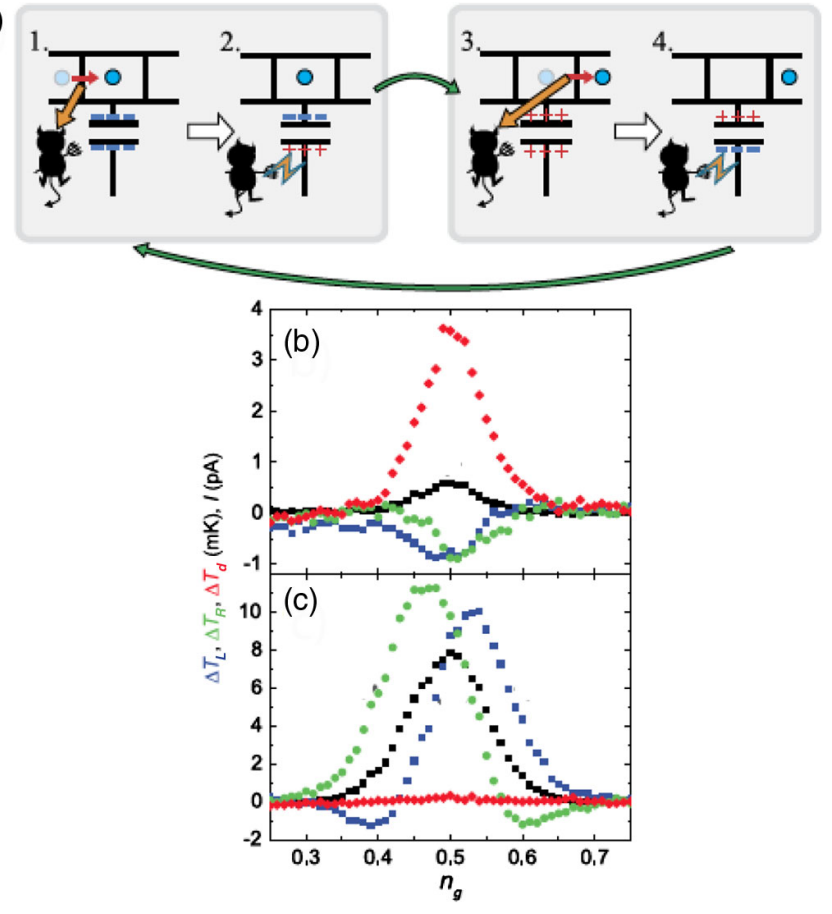

FIG. 15. (a) Principle of the experimental realization of the autonomous Maxwell's demon. The horizontal top row schematizes a single-electron transistor. Electrons (blue circle) can tunnel inside the central island from the left wall and outside from the right wall. The demon watches at the state of the island and it applies a positive charge to attract the electrons when they tunnel inside and repels them when they tunnel outside. The systems cools because of the energy released toward the heat bath by the tunneling events, and the presence of the demon makes the cooling processes more efficient. The energy variation of the processes is negative because of the information introduced by the demon. (b) The measured temperature variations of the left (blue line) and right (green line) leads as a function of the external control parameter $n_{g}$ when the demon is active and the bath temperature is $50 \mathrm{mK}$. We see that at the optimum value, $n_{g}=1 / 2$, both leads are cooled to about $1 \mathrm{mK}$ and the current $I$ flowing through the SET (black line) has a maximum. At the same time, in order to processes information the temperature of the demon (red line) increases a few $\mathrm{mK}$. (c) The same parameters of (b) are measured when the demon is not active. We see that the demon temperature does not change, whereas both leads are now heated by the current $I$.

Thus, the total (system plus demon) energy production is positive. The coupling of the demon with the SET can be controlled by a second gate which acts on the singleelectron box. In Fig. 15(c) we plot the measured temperatures when the demon has been switched off. We clearly see that in such a case the demon temperature does not change and the two electrodes are heating up because of the current flow. As far as I know, this is the only example that shows that under specific conditions an autonomous local Maxwell's demon, which does not use the external feedback, can be realized.

\section{B. Energy cost of information erasure}

The experiments in the previous two sections show that one can extract work from information. In the rest of this section, we discuss the reverse process, i.e., the energy needed to erase information. By applying the second law of thermodynamics, Landauer demonstrated that information erasure is necessarily a dissipative process: the erasure of one bit of information is accompanied by the production of at least $k_{B} T \ln (2)$ of heat into the environment. This result is known as Landauer's erasure principle. It emphasizes the fundamental difference between the process of writing and erasing information. Writing is akin to copying information from one device to another: state left is mapped to left and state right is mapped to right, for example. This one-to-one mapping can be realized in principle without dissipating any heat (in statistical mechanics one would say that it conserves the volume in phase space). By contrast, erasing information is a two-to-one transformation: states left and right are mapped onto one single state, say, right (this process does not conserve the volume in phase space and is thus dissipative).

Landauer's original thought experiment was realized $[150,151]$ for the first time in a real system in 2011 using a colloidal Brownian particle in a fluid trapped in a doublewell potential produced by two strongly focused laser beams. This system has two distinct states (particle in the right or left well) and may thus be used to store one bit of information. The erasure principle has been verified by implementing a protocol proposed by Bennett and illustrated in Fig. 16. At the beginning of the erasure process, the colloidal particle may be either in the left or right well with equal probability of one-half. The erasure protocol is composed of the following steps: (1) the barrier height is first decreased by varying the laser intensity, (2) the particle is then pushed to the right by gently inclining the potential. and (3) the potential is brought back to its initial shape. At the end of the process, the particle is in the right well with unit probability, irrespective of its departure position. As in the previous experiment, the position of the particle is recorded with the help of a camera. For a full erasure cycle, the average heat dissipated into the environment is equal to the average work needed to modulate the form of the double-well potential. This quantity was evaluated from the measured trajectory and shown to always be larger than the Landauer bound, which is asymptotically approached in the limit of long erasure times. However, in order to reach the bound, the protocol must be accurately chosen because, as discussed in Ref. [150] and shown experimentally [152], there are protocols that are intrinsically irreversible no matter how slowly they are performed. The way in which a protocol can be optimized has been theoretically solved in Ref. [153], but the optimal protocol is not often easy to apply in an experiment. 

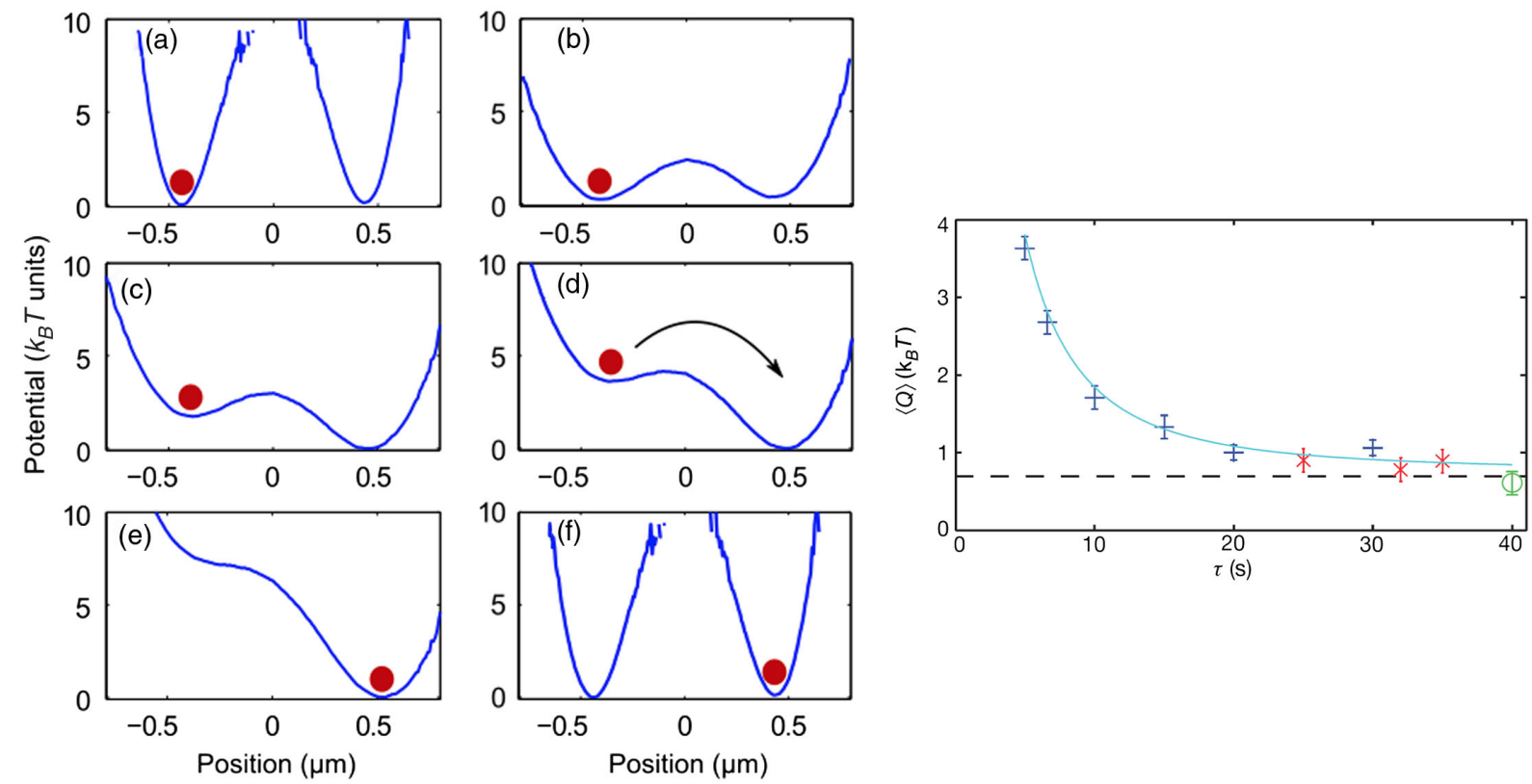

FIG. 16. Experimental verification of Landauer's erasure principle. A colloidal particle is initially confined in one of two wells of a double-well potential with probability one-half. This configuration stores one bit of information. By modulating the height of the barrier and applying a tilt, the particle can be brought to one of the wells with probability one, irrespective of the initial position. This final configuration corresponds to zero bit of information. In the limit of long erasure cycles, the heat dissipated during the erasure process can approach, but not exceed, the Landauer bound indicated by the dashed line in the right panel (see Ref. [150] for details).

\section{Other examples on the connection between information and energy}

By having successfully turned gedanken into real experiments, the above three seminal examples provide a firm empirical foundation to the physics of information and the intimate connection existing between information and energy. This connection is reenforced by the relationship between the generalized Jarzinsky equality [154] and the Landauer bound, which has been proved and tested on experimental data in Ref. [151]. A recent article [155] extends the equivalence of information and thermodynamic entropies at thermal equilibrium.

A number of additional experiments have been performed on this subject [156-160]. For example, in Ref. [157] the symmetry breaking, induced in the probability distribution of the position of a Brownian particle, is studied by commuting the trapping potential from a single- to a double-well potential. The authors measured the time evolution of the system entropy and showed how to produce work from information. The experiment in Ref. [160] shows that using a Maxwell's demon in the information erasure costs less energy than the Landauer bound. It is worth mentioning experiments where the Landauer bound has been reached in nano devices $[156,159]$. These experiments open the way to insightful applications for future developments of information technology.

Finally, the connection between thermodynamics and information plays a very important role in the understanding of biological systems [161,162]. Indeed, information can be used to produce motion and, on the contrary, information processing needs energy. We do not discuss in more detail this important topic, which is developed in two other articles [163,164].

\section{USE OF STOCHASTIC THERMODYNAMICS IN EXPERIMENTS: DISCUSSION AND PERSPECTIVES}

In this review we present various experimental results that allow us to introduce several fundamental concepts of stochastic thermodynamics, such as the FT for heat and work, the Jarzynski equality, and the trajectory entropy. We have already mentioned several applications of these concepts to the measure of the system response in NESS, to the free-energy estimation, and to the relationship between information and thermodynamics. In the experiments that we have described, all of the theoretical predictions are perfectly verified; thus, the question here is to see whether those findings of stochastic thermodynamics might become a useful measurement tool that allows us to calibrate and to make predictions in experiments. We discuss here several examples.

\section{A. Using FT to calibrate an experiment}

FT can be used to have a precise estimation of either an offset or a calibration error in an experiment. The method can be easily understood by considering the systems modeled by either one or several Langevin 
equations described in Secs. II, III D, and V. For these systems we have seen that the work and heat PDF satisfy a FT. Thus, in an experimental system that can be modeled by the Langevin equation, one can check the good calibration of the apparatus by computing the work and checking whether the FT holds for the measured variables. If it does not hold, it means that some error has been made in the calibration or some small offset exists in the measured quantities (see also Refs. [38,165]).

\section{B. Role of hidden variables}

Along the same line of Sec. IX A, FT can be used to estimate unknown parameters of a device. For example, such a method has been used to measure the power of molecular motors $[38,166]$. The idea is certainly very smart and merits serious consideration for applications. However, one has to pay attention to the influence of hidden variables. Indeed, in Ref. [167] it has been pointed out that in the abovementioned experiment of Ref. [166] a hidden variable has been neglected; thus, the estimated value of the motor power could be affected by a large error. Another interesting analysis on hidden variables has been done in Ref. [168] for the experiment of Ref. [169] on a SET. In this experiment the bias obtained from the measured FT does not completely match with the experimental value of the applied bias. In Ref. [168] it has been shown that this discrepancy is due to the additional bias introduced by the SET that is used to measure the electron transfers. More recent measures proved that this is the case [170]. Another accurate experimental analysis of the role of a hidden variable on FT properties has been reported in Ref. [171]. This role can be easily understood by looking at the experiment described in Sec. V, which is modeled by the coupled Langevin equations (20) and (21). Suppose now that in that experiment instead of having access to both $V_{1}$ and $V_{2}$ variables, only one of the two can be measured. We immediately see that there is no way of extracting the good values from the measurements. Thus, the use of FT to extract experimental parameters from an experiment might be a very good method, but it has to be used with caution because of the possible existence of hidden variables.

\section{Statistical inferences}

The problem of hidden variables mentioned in Sec. IX B has been attacked in a different way in Refs. [172,173]. This approach, called statistical inferences by the authors, analyzes to what extent the fact that FT and FDR do not hold can give information on hidden variables. In Ref. [173] the authors have been able to extract interesting information on a single-molecule measurements. Thus, the approach is certainly interesting and it merits further study in more detail in the future.

\section{Nano or micro motor efficiency}

The other aspect that we have briefly discussed in Sec. VI concerns the efficiency of nano or micro motor. In spite of the large number of theoretical results on this subject, there is a lack of experiments in this important field, especially in what concerns the efficiency of power supplies and of chemically driven motors. A bound on the efficiency of these motors has been recently fixed by the recently discovered indetermination relations [114-116]. However, as we have already said, no proof-of-principle experiment, such as the Carnot cycle discussed in Sec. VI, has been performed for chemically driven motors.

\section{E. Role of Maxwell's demons}

The role of Maxwell's demons in increasing the efficiency of small devices is certainly very important. This subject is in its infancy and it might have very powerful applications. The described autonomous Maxwell's demon is certainly a smart system. However, it works because of the very small operational temperature of the device. A very big challenge is the realization of such an autonomous demon for a device working at room temperature. At the moment, such a device does not exist, and it is not even clear in which physical or chemical system it could be realized.

\section{F. Energy information connection}

The connection of stochastic thermodynamics with the energy dissipation in each logic operation is the last issue that we have developed. It is clearly very important in connection to the autonomous Maxwell's demon to estimate the amount of work that the demon has to perform in order to process information. This will allow us to decide whether the application of a demon is really an advantage to reduce the energy consumption. Furthermore, deeper knowledge of the connection between thermodynamics and information is certainly useful not only to understand biological processes but also to develop methods that allow us to recover energy during reversible logical operation. Also, this field is in his infancy and future development will certainly appear.

\section{G. Macroscopic and self-propelling systems}

The are several aspects that we do not present because this will extend too much the purpose of this review. The first is the application to quantum systems, which presents several problems discussed in another article [174]. The other aspects are those related to the application of stochastic thermodynamics to dissipative chaotic systems driven out of equilibrium by external forces such as, for example, shaken granular media, turbulence fields, and chaotic nonlinear oscillators. In these systems the main source of randomness is not the thermal noise but the chaotic behavior produced by the complex dynamics. As summarized in Ref. [3], the main problem is the absence of 
a characteristic energy scale for all the degrees of freedom, something similar to an "effective temperature." Thus, the possibility of applying the same tools discussed in this article to nonthermal systems is certainly not universal and it has to be studied in the specific cases. The same problem occurs in the more recent applications of the stochastic thermodynamics tools to the fluctuations in self-propelling systems [32,33], on which it is difficult to make very general statements as for the systems analyzed in this article.

\section{H. Conclusions}

This review of the experimental aspects and applications of stochastic thermodynamics is certainly not exhaustive, as several topics have only been mentioned and not developed. Furthermore, quantum aspects and macroscopic systems have not been treated at all. However, we think that this review gives a reasonable idea of what has been already done, of what remains to do, and of what the possible practical applications of stochastic thermodynamics are.

\section{ACKNOWLEDGMENTS}

We thank I. Martinez, J. Pekola, and F. Ritort for useful comments and their permission to use the figures of their articles. We acknowledge useful discussion with M. Esposito. This work has been partially supported by ERC contract OUTEFLUCOP.

[1] K. Sekimoto, Stochastic Energetics, Lecture Notes in Physics, Vol. 799 (Springer, New York, 2010).

[2] U. Seifert, Stochastic Thermodynamics, Fluctuation Theorems and Molecular Machines, Rep. Prog. Phys. 75, 126001 (2012).

[3] S. Ciliberto, R. Gomez-Solano, and A. Petrosyan, Fluctuations, Linear Response, and Currents in Out-ofEquilibrium Systems, Annu. Rev. Condens. Matter Phys. 4, 235 (2013).

[4] D. J. Evans, E. G. D. Cohen, and G. P. Morriss, Probability of Second Law Violations in Shearing Steady States, Phys. Rev. Lett. 71, 2401 (1993).

[5] D. J. Evans and D. J. Searles, The Fluctuation Theorem, Adv. Phys. 51, 1529 (2002).

[6] G. Gallavotti and E. G. D. Cohen, Dynamical Ensembles in Nonequilibrium Statistical Mechanics, Phys. Rev. Lett. 74, 2694 (1995).

[7] J. L. Lebowitz and H. Spohn, A Gallavotti-Cohen-Type Symmetry in the Large Deviation Functional for Stochastic Dynamics, J. Stat. Phys. 95, 333 (1999).

[8] J. Kurchan, Fluctuation Theorem for Stochastic Dynamics, J. Phys. A 31, 3719 (1998).

[9] D. J. Searles, L. Rondoni, and D. J. Evans, The Steady State Fluctuation Relation for the Dissipation Function, J. Stat. Phys. 128, 1337 (2007).

[10] D. J. Evans, D. J. Searles, and S. R. Williams, On the Fluctuation Theorem for the Dissipation Function and Its
Connection with Response Theory, J. Chem. Phys. 128, 014504 (2008).

[11] U. Seifert, Entropy Production along a Stochastic Trajectory and an Integral Fluctuation Theorem, Phys. Rev. Lett. 95, 040602 (2005).

[12] L Puglisi, A. Rondoni, and A Vulpiani, Relevance of Initial and Final Conditions for the Fluctuation Relation in Markov Processes, J. Stat. Mech. (2006) P08010.

[13] C. Jarzynski, Nonequilibrium Equality for Free Energy Differences, Phys. Rev. Lett. 78, 2690 (1997).

[14] C. Jarzynski, Equilibrium Free-Energy Differences from Nonequilibrium Measurements: A Master-Equation Approach, Phys. Rev. E 56, 5018 (1997).

[15] G. E. Crooks, Nonequilibrium Measurements of Free Energy Differences for Microscopically Reversible Markovian Systems, J. Stat. Phys. 90, 1481 (1998).

[16] T. Speck and U. Seifert, Extended Fluctuation-Dissipation Theorem for Soft Matter in Stationary Flow, Phys. Rev. E 79, 040102 (2009).

[17] U. Seifert and T. Speck, Fluctuation-Dissipation Theorem in Nonequilibrium Steady States, Europhys. Lett. 89, 10007 (2010).

[18] J. R. Gomez-Solano, A. Petrosyan, S. Ciliberto, R. Chetrite, and K. Gawędzki, Experimental Verification of a Modified Fluctuation-Dissipation Relation for a Micron-Sized Particle in a Nonequilibrium Steady State, Phys. Rev. Lett. 103, 040601 (2009).

[19] J. Prost, J.-F. Joanny, and J. M. Parrondo, Generalized Fluctuation-Dissipation Theorem for Steady-State Systems, Phys. Rev. Lett. 103, 090601 (2009).

[20] M. Baiesi, C. Maes, and B. Wynants, Fluctuations and Response of Nonequilibrium States, Phys. Rev. Lett. 103, 010602 (2009).

[21] M. Baiesi, S. Ciliberto, G. Falasco, and C. Yolcu, Thermal Response of Nonequilibrium RC Circuits, Phys. Rev. E 94, 022144 (2016).

[22] L. F. Cugliandolo, J. Kurchan, and L. Peliti, Energy Flow, Partial Equilibration, and Effective Temperatures in Systems with Slow Dynamics, Phys. Rev. E 55, 3898 (1997).

[23] L. Berthier, P. C. W. Holdsworth, and M. Sellitto, Nonequilibrium Critical Dynamics of the Two-Dimensional XY Model, J. Phys. A 34, 1805 (2001).

[24] L. F. Cugliandolo, The Effective Temperature, J. Phys. A 44, 483001 (2011)

[25] R. Labbé, J.-F. Pinton, and S. Fauve, Power Fluctuations in Turbulent Swirling Flows, J. Phys. II (France) 6, 1099 (1996).

[26] S. Ciliberto, N. Garnier, S. Hernandez, C. Lacpatia, J.-F. Pinton, and G. Ruiz Chavarria, Experimental Test of the Gallavotti-Cohen Fluctuation Theorem in Turbulent Flows, Physica (Amsterdam) 340A, 240 (2004).

[27] K. Feitosa and N. Menon, Fluidized Granular Medium as an Instance of the Fluctuation Theorem, Phys. Rev. Lett. 92, 164301 (2004).

[28] N. Kumar, S. Ramaswamy, and A. K. Sood, Symmetry Properties of the Large-Deviation Function of the Velocity of a Self-Propelled Polar Particle, Phys. Rev. Lett. 106, 118001 (2011).

[29] Anne Mounier and Antoine Naert, The Hatano-Sasa Equality: Transitions between Steady States in a Granular Gas, Europhys. Lett. 100, 30002 (2012). 
[30] A. Naert, Experimental Study of Work Exchange with a Granular Gas: The Viewpoint of the Fluctuation Theorem, Europhys. Lett. 97, 20010 (2012).

[31] O. Cadot, A. Boudaoud, and C. Touzé, Statistics of Power Injection in a Plate Set into Chaotic Vibration, Eur. Phys. J. B 66, 399 (2008).

[32] A. Argun, A.-R. Moradi, E. Pince, G. B. Bagci, and G. Volpe, Experimental Evidence of the Failure of Jarzynski Equality in Active Baths, arXiv:1601.01123.

[33] S. Krishnamurthy, S. Ghosh, D. Chatterji, R. Ganapathy, and A. K. Sood, A Micrometre-Sized Heat Engine Operating between Bacterial Reservoirs, Nat. Phys. 12, 1134 (2016).

[34] U. Marini Bettolo Marconi, A. Puglisi, L. Rondoni, and A. Vulpiani, Fluctuation-Dissipation: Response Theory in Statistical Physics, Phys. Rep. 461, 111 (2008).

[35] A. Kis Andras and A. Zettl, Nanomechanics of Carbon Nanotubes, Phil. Trans. R. Soc. A 366, 1591 (2008).

[36] J. R. Gomez-Solano, L. Bellon, A. Petrosyan, and S. Ciliberto, Steady-State Fluctuation Relations for Systems Driven by an External Random Force, Europhys. Lett. 89, 60003 (2010).

[37] L. Bellon, L. Buisson, S. Ciliberto, and F. Vittoz, Zero Applied Stress Rheometer, Rev. Sci. Instrum. 73, 3286 (2002).

[38] S. Ciliberto, S. Joubaud, and A Petrosian, Fluctuations in Out of Equilibrium Systems: From Theory to Experiment, J. Stat. Mech. (2010) P12003.

[39] L. Bellon, S. Ciliberto, H. Boubaker, and L. Guyon, Differential Interferometry with a Complex Contrast, Opt. Commun. 207, 49 (2002).

[40] F. Douarche, S. Ciliberto, and Petrosyan, Estimate of the Free Energy Difference in Mechanical Systems from Work Fluctuations: Experiments and Models, J. Stat. Mech. (2005) P09011.

[41] F. Douarche, S. Joubaud, N. B. Garnier, A. Petrosyan, and S. Ciliberto, Work Fluctuation Theorems for Harmonic Oscillators, Phys. Rev. Lett. 97, 140603 (2006).

[42] F. Douarche, L Buisson, S Ciliberto, and A Petrosyan, A Simple Noise Subtraction Technique, Rev. Sci. Instrum. 75, 5084 (2004).

[43] S. Joubaud, N. B. Garnier, and S. Ciliberto, Fluctuation Theorems for Harmonic Oscillators, J. Stat. Mech. (2007) P09018.

[44] K. Sekimoto, Langevin Equation and Thermodynamics, Prog. Theor. Phys. Suppl. 130, 17 (1998).

[45] V. Blickle, T. Speck, L. Helden, U. Seifert, and C. Bechinger, Thermodynamics of a Colloidal Particle in a Time-Dependent Nonharmonic Potential, Phys. Rev. Lett. 96, 070603 (2006).

[46] S. Schuler, T. Speck, C. Tietz, J. Wrachtrup, and U. Seifert, Experimental Test of the Fluctuation Theorem for a Driven Two-Level System with Time-Dependent Rates, Phys. Rev. Lett. 94, 180602 (2005).

[47] F. Zamponi, F. Bonetto, L. F. Cugliandolo, and J. Kurchan, A Fluctuation Theorem for Non-Equilibrium Relaxational Systems Driven by External Forces, J. Stat. Mech. (2005) P09013, 2005.

[48] R. van Zon, S. Ciliberto, and E. G. D. Cohen, Power and Heat Fluctuation Theorems for Electric Circuits, Phys. Rev. Lett. 92, 130601 (2004).
[49] R. van Zon and E. G. D. Cohen, Extension of the Fluctuation Theorem, Phys. Rev. Lett. 91, 110601 (2003).

[50] P. Gaspard, Time-Reversed Dynamical Entropy and Irreversibility in Markovian Random Processes, J. Stat. Phys. 117, 599 (2004).

[51] D. Andrieux, P. Gaspard, S. Ciliberto, S. Garnier, S. Joubaud, and S. Petrosyan, Thermodynamic Time Asymmetry in Non-Equilibrium Fluctuations, J. Stat. Mech. (2008) P01002.

[52] S. Joubaud, N. B. Garnier, and S. Ciliberto, Fluctuations of the Total Entropy Production in Stochastic Systems, Europhys. Lett. 82, 30007 (2008).

[53] M. Esposito and C. Van den Broeck, Three Detailed Fluctuation Theorems, Phys. Rev. Lett. 104, 090601 (2010).

[54] G. Bulnes Cuetara, M. Esposito, and A. Imparato, Exact Fluctuation Theorem without Ensemble Quantities, Phys. Rev. E 89, 052119 (2014).

[55] T. Speck and U. Seifert, Dissipated Work in Driven Harmonic Diffusive Systems: General Solution and Application to Stretching Rouse Polymers, Eur. Phys. J. B 43, 521 (2005).

[56] P. Jop, A. Petrosyan, and S. Ciliberto, Work and Dissipation Fluctuations Near the Stochastic Resonance of a Colloidal Particle, Europhys. Lett. 81, 50005 (2008).

[57] R. Benzi, G. Parisi, A. Sutera, and A. Vulpiani, A Theory of Stochastic Resonance in Climatic Change, SIAM J. Appl. Math. 43, 565 (1983).

[58] L. Gammaitoni, F. Marchesoni, and S. Santucci, Stochastic Resonance as a Bona Fide Resonance, Phys. Rev. Lett. 74, 1052 (1995).

[59] C. Schmitt, B. Dybiec, P. Hänggi, and C. Bechinger, Stochastic Resonance vs. Resonant Activation, Europhys. Lett. 74, 937 (2006).

[60] A. Imparato, P. Jop, A. Petrosyan, and S. Ciliberto, Probability Density Functions of Work and Heat Near the Stochastic Resonance of a Colloidal Particle, J. Stat. Mech. (2008) P10017, 2008.

[61] V. Blickle, T. Speck, C. Lutz, U. Seifert, and C. Bechinger, Einstein Relation Generalized to Nonequilibrium, Phys. Rev. Lett. 98, 210601 (2007).

[62] A. Imparato and L. Peliti, Work Distribution and Path Integrals in General Mean-Field Systems, Europhys. Lett. 70, 740 (2005).

[63] C. Jarzynski, Equilibrium Free-Energy Differences from Nonequilibrium Measurements: A Master-Equation Approach, Annu. Rev. Condens. Matter Phys. 2, 329 (2011).

[64] J. Liphardt, S. Dumont, S. B. Smith, and I. Tinoco, Jr., and C. Bustamante, Equilibrium Information from Nonequilibrium Measurements in an Experimental Test of Jarzynski's Equality, Science 296, 1832 (2002).

[65] A. Mossa, M. Manosas, N. Forms, J. M. Huguet, and Felix Ritort, Dynamic Force Spectroscopy of DNA Hairpins: I. Force Kinetics and Free Energy Landscapes, J. Stat. Mech. (2009) P02060.

[66] C. Bustamante, J. Liphardt, and F. Ritort, The Nonequilibrium Thermodynamics of Small Systems, Phys. Today 58, No. 7, 43 (2005).

[67] D. Collin, F. Ritort, C. Jarzynski, S. B. Smith, I. Tinoco, and C. Bustamante, Verification of the Crooks Fluctuation 
Theorem and Recovery of RNA Folding Free Energies, Nature (London) 437, 231 (2005).

[68] G. Hummer and A. Szabo, Free Energy Profiles from Single-Molecule Pulling Experiments, Proc. Natl. Acad. Sci. U.S.A. 107, 21441 (2010).

[69] A. Alemany, A. Mossa, I. Junier, and F. Ritort, Experimental Free-Energy Measurements of Kinetic Molecular States Using Fluctuation Theorems, Nat. Phys. 8, 688 (2012).

[70] D. Jeong and I. Andricioaei, Reconstructing Equilibrium Entropy and Enthalpy Profiles from Non-Equilibrium Pulling, J. Chem. Phys. 138, 114110 (2013).

[71] S. Raman, T. Utzig, T. Baimpos, B. R. Shrestha, and M. Valtiner, Deciphering the Scaling of Single-Molecule Interactions Using Jarzynski's Equality, Nat. Commun. 5, 5539 (2014).

[72] A. N. Gupta, A. Vincent, K. Neupane, H. Yu, F. Wang, and M. T. Woodside, Experimental Validation of Free-EnergyLandscape Reconstruction from Non-Equilibrium SingleMolecule Force Spectroscopy Measurements, Nat. Phys. 7, 631 (2011).

[73] W.-S. Chen, W.-H. Chen, Z. Chen, A. A. Gooding, K.-J. Lin, and C.-H. Kiang, Direct Observation of Multiple Pathways of Single-Stranded DNA Stretching, Phys. Rev. Lett. 105, 218104 (2010).

[74] E. W. Frey, J. Li, S. S. Wijeratne, and C.-H. Kiang, Reconstructing Multiple Free Energy Pathways of DNA Stretching from Single Molecule Experiments, J. Phys. Chem. B 119, 5132 (2015).

[75] M. C. Engel, D. B. Ritchie, D. A. N. Foster, K. S. D. Beach, and M. T. Woodside, Reconstructing Folding Energy Landscape Profiles from Nonequilibrium Pulling Curves with an Inverse Weierstrass Integral Transform, Phys. Rev. Lett. 113, 238104 (2014).

[76] A. P. Manuel, J. Lambert, and M. T. Woodside, Reconstructing Folding Energy Landscapes from Splitting Probability Analysis of Single-Molecule Trajectories, Proc. Natl. Acad. Sci. U.S.A. 112, 7183 (2015).

[77] J. Camunas-Soler, A. Alemany, and F. Ritort, Experimental Measurement of Binding Energy, Selectivity, and Allostery Using Fluctuation Theorems, Science 355, 412 (2017).

[78] T. Hatano and S.-i. Sasa, Steady-State Thermodynamics of Langevin Systems, Phys. Rev. Lett. 86, 3463 (2001).

[79] E. H. Trepagnier, C. Jarzynski, F. Ritort, G. E. Crooks, C. J. Bustamante, and J. Liphardt, Experimental Test of Hatano and Sasa's Nonequilibrium Steady-State Equality, Proc. Natl. Acad. Sci. U.S.A. 101, 15038, 15038 (2004).

[80] T. Bodineau and B. Derrida, Current Fluctuations in Nonequilibrium Diffusive Systems: An Additivity Principle, Phys. Rev. Lett. 92, 180601 (2004).

[81] C. Jarzynski and D. K. Wójcik, Classical and Quantum Fluctuation Theorems for Heat Exchange, Phys. Rev. Lett. 92, 230602 (2004).

[82] C. Van den Broeck, R. Kawai, and P. Meurs, Microscopic Analysis of a Thermal Brownian Motor, Phys. Rev. Lett. 93, 090601 (2004).

[83] P. Visco, Work Fluctuations for a Brownian Particle between Two Thermostats, J. Stat. Mech. (2006) P06006.
[84] D. J. Evans, D. J. Searles, and S. R. Williams, On the Probability of Violations of Fourier's Law for Heat Flow in Small Systems Observed for Short Times, J. Chem. Phys. 132, 024501 (2010).

[85] A. Crisanti, A. Puglisi, and D. Villamaina, Nonequilibrium and Information: The Role of Cross Correlations, Phys. Rev. E 85, 061127 (2012).

[86] A. Sarracino, D. Villamaina, G. Gradenigo, and A. Puglisi, Irreversible Dynamics of a Massive Intruder in Dense Granular Fluids, Europhys. Lett. 92, 34001 (2010).

[87] V. Dotsenko, A. Maciołek, O. Vasilyev, and G. Oshanin, Two-Temperature Langevin Dynamics in a Parabolic Potential, Phys. Rev. E 87, 062130 (2013).

[88] H. C. Fogedby and A. Imparato, A Bound Particle Coupled to Two Thermostats, J. Stat. Mech. (2011) P05015.

[89] H. C. Fogedby and A. Imparato, Heat Flow in Chains Driven by Thermal Noise, J. Stat. Mech. (2012) P04005.

[90] H. C. Fogedby and A. Imparato, Heat Fluctuations and Fluctuation Theorems in the Case of Multiple Reservoirs, J. Stat. Mech. (2014) P11011.

[91] S. Ciliberto, A. Imparato, A. Naert, and M. Tanase, Heat Flux and Entropy Produced by Thermal Fluctuations, Phys. Rev. Lett. 110, 180601 (2013).

[92] S. Ciliberto, A. Imparato, A. Naert, and M. Tanase, Statistical Properties of the Energy Exchanged between Two Heat Baths Coupled by Thermal Fluctuations, J. Stat. Mech. (2013) P12014.

[93] J. V. Koski, T. Sagawa, O. P. Saira, Y. Yoon, A. Kutvonen, P. Solinas, M. Möttönen, T. Ala-Nissila, and J. P. Pekola, Distribution of Entropy Production in a Single-Electron Box, Nat. Phys. 9, 644 (2013).

[94] A. Bérut, A. Petrosyan, and S. Ciliberto, Energy Flow between Two Hydrodynamically Coupled Particles Kept at Different Effective Temperatures, Europhys. Lett. 107, 60004 (2014).

[95] A. Bérut, A. Imparato, A. Petrosyan, and S. Ciliberto, Stationary and Transient Fluctuation Theorems for Effective Heat Flux between Hydrodynamically Coupled Particles in Optical Traps, Phys. Rev. Lett. 116, 068301 (2016).

[96] G. Cannata, G. Scandurra, and C. Ciofi, An Ultralow Noise Preamplifier for Low Frequency Noise Measurements, Rev. Sci. Instrum. 80, 114702 (2009).

[97] A. Bérut, A. Imparato, A. Petrosyan, and S. Ciliberto, The Role of Coupling on the Statistical Properties of the Energy Fluxes between Stochastic Systems at Different Temperatures, J. Stat. Mech. (2016) P054002.

[98] A. Bérut, A. Imparato, A. Petrosyan, and S. Ciliberto, Theoretical Description of Effective Heat Transfer between Two Viscously Coupled Beads, Phys. Rev. E 94, 052148 (2016).

[99] D. V. Averin and J. P. Pekola, Statistics of the Dissipated Energy in Driven Single-Electron Transitions, Europhys. Lett. 96, 67004 (2011).

[100] J. P. Pekola, A. Kutvonen, and T. Ala-Nissila, Dissipated Work and Fluctuation Relations for Non-Equilibrium Single-Electron Transitions, J. Stat. Mech. (2013) P02033.

[101] D.S. Golubev and J.P. Pekola, Statistics of Heat Exchange between Two Resistors, Phys. Rev. B 92, 085412 (2015). 
[102] M. Esposito, R. Kawai, K. Lindenberg, and C. Van den Broeck, Efficiency at Maximum Power of Low-Dissipation Carnot Engines, Phys. Rev. Lett. 105, 150603 (2010).

[103] T. Schmiedl and U. Seifert, Efficiency at Maximum Power: An Analytically Solvable Model for Stochastic Heat Engines, Europhys. Lett. 81, 20003 (2008).

[104] M. Polettini, G. Verley, and M. Esposito, Efficiency Statistics at All Times: Carnot Limit at Finite Power, Phys. Rev. Lett. 114, 050601 (2015).

[105] G. Verley, T. Willaert, C. Van den Broeck, and M. Esposito, Universal Theory of Efficiency Fluctuations, Phys. Rev. E 90, 052145 (2014).

[106] G. Verley, T. Willaert, C. Van den Broeck, and M. Esposito, The Unlikely Carnot Efficieny, Nat. Commun. 5, 4721 (2014).

[107] E. Zimmermann and U. Seifert, Efficiencies of a Molecular Motor: A Generic Hybrid Model Applied to the $\mathrm{F}_{1}$ ATPase, New J. Phys. 14, 103023 (2012).

[108] V. Blickle and C. Bechinger, Realization of a MicrometreSized Stochastic Heat Engine, Nat. Phys. 8, 143 (2012).

[109] I. A. Martinez, E. Roldán, L. Dinis, D. Petrov, I. A. Parrondo, and R. A. Rica, Brownian Carnot Engine, Nat. Phys. 12, 67 (2016).

[110] M. Serra-Garcia, A. Foehr, M. Molerón, J. Lydon, C. Chong, and C. Daraio, Mechanical Autonomous Stochastic Heat Engine, Phys. Rev. Lett. 117, 010602 (2016).

[111] J. Ronagel, S. T. Dawkins, K. N. Tolazzi, O. Abah, E. Lutz, F. Schmidt-Kaler, and K. Singer, A Single-Atom Heat Engine, Science 352, 325 (2016).

[112] I. A. Martinez, E. Roldán, L. Dinis, D. Petrov, and R. A. Rica, Adiabatic Processes Realized with a Trapped Brownian Particle, Phys. Rev. Lett. 114, 120601 (2015).

[113] K. Proesmans, Y. Dreher, M. Gavrilov, J. Bechhoefer, and C. Van den Broeck, Brownian Duet: A Novel Tale of Thermodynamic Efficiency, Phys. Rev. X 6, 041010 (2016).

[114] A. C. Barato and U. Seifert. Thermodynamic Uncertainty Relation for Biomolecular Processes, Phys. Rev. Lett. 114, 158101 (2015).

[115] P. Pietzonka, A. C. Barato, and U. Seifert, Universal Bound on the Efficiency of Molecular Motors, J. Stat. Mech. (2016) P124004.

[116] T. R. Gingrich, J. M. Horowitz, N. Perunov, and J. L. England, Dissipation Bounds All Steady-State Current Fluctuations, Phys. Rev. Lett. 116, 120601 (2016).

[117] P. Reimann, C. Van den Broeck, H. Linke, P. Hänggi, J. M. Rubi, and A. Pérez-Madrid, Giant Acceleration of Free Diffusion by Use of Tilted Periodic Potentials, Phys. Rev. Lett. 87, 010602 (2001).

[118] T.S. Grigera and N.E. Israeloff, Observation of Fluctuation-Dissipation-Theorem Violations in a Structural Glass, Phys. Rev. Lett. 83, 5038 (1999).

[119] L. Bellon, S. Ciliberto, and C. Laroche, Violation of the Fluctuation-Dissipation Relation during the Formation of a Colloidal Glass, Europhys. Lett. 53, 511 (2001).

[120] D. Herisson and M. Ocio, Fluctuation-Dissipation Ratio of a Spin Glass in the Aging Regime, Phys. Rev. Lett. 88, 257202 (2002).

[121] L. Berthier and J.-L. Barrat, Shearing a Glassy Material: Numerical Tests of Nonequilibrium Mode-Coupling
Approaches and Experimental Proposals, Phys. Rev. Lett. 89, 095702 (2002).

[122] A. Crisanti and J. Ritort, Violation of the FluctuationDissipation Theorem in Glassy Systems: Basic Notions and the Numerical Evidence, J. Phys. A 36, R181 (2003).

[123] P. Calabrese and A. Gambassi, Ageing Properties of Critical System, J. Phys. A 38, R133 (2005).

[124] A. Barrat, V. Colizza, and V. Loreto, FluctuationDissipation Ratio for Compacting Granular Media, Phys. Rev. E 66, 011310 (2002).

[125] K. Hayashi and M. Takano, Abstract Image Violation of the Fluctuation-Dissipation Theorem in a Protein System, Biophys. J. 93, 895 (2007).

[126] L. Conti, P. DeGregeorio, M. Bonaldi, A. Borrielli, M. Crivellari, G. Karapetyan, C. Poli, E. Serra, R. K. Thakur, and L. Rondoni, Elasticity of Mechanical Oscillators in Nonequilibrium Steady States: Experimental, Numerical, and Theoretical Results, Phys. Rev. E 85, 066605 (2012).

[127] P. Hänggi and H. Thomas, Linear Response and Fluctuation Theorems for Nonstationary Stochastic Processes, Z. Phys. 22, 295 (1975).

[128] T. Harada and S.-i. Sasa, Equality Connecting Energy Dissipation with a Violation of the Fluctuation-Response Relation, Phys. Rev. Lett. 95, 130602 (2005).

[129] E. Lippiello, F. Corberi, and M. Zannetti, Off-Equilibrium Generalization of the Fluctuation Dissipation Theorem for Ising Spins and Measurement of the Linear Response Function, Phys. Rev. E 71, 036104 (2005).

[130] T. Speck and U. Seifert, Restoring a FluctuationDissipation Theorem in a Nonequilibrium Steady State, Europhys. Lett. 74, 391 (2006).

[131] R. Chetrite, G. Falkovich, and K. Gawędzki, Fluctuation Relations in Simple Examples of Non-Equilibrium Steady States, J. Stat. Mech. (2008) P08005.

[132] R. Chetrite and S. Gupta, Two Refreshing Views of Fluctuation Theorems through Kinematics Elements and Exponential Martingale, J. Stat. Phys. 143, 543 (2011).

[133] M. Baiesi, C. Maes, and B. Wynants, Nonequilibrium Linear Response for Markov Dynamics, I: Jump Processes and Overdamped Diffusions, J. Stat. Phys. 137, 1094 (2009).

[134] K. Hayashi and S. Sasa, Effective Temperature in Nonequilibrium Steady States of Langevin Systems with a Tilted Periodic Potential, Phys. Rev. E 69, 066119 (2004).

[135] T. Sakaue and T. Ohta, DNA Methylation is Dispensable for the Growth and Survival of the Extraembryonic Lineages, Phys. Rev. E 77, 050102(R) (2008).

[136] B. Altaner, M. Polettini, and M. Esposito, FluctuationDissipation Relations Far from Equilibrium, Phys. Rev. Lett. 117, 180601 (2016).

[137] J. Mehl, V. Blickle, U. Seifert, and C. Bechinger, Experimental Accessibility of Generalized FluctuationDissipation Relations for Nonequilibrium Steady States, Phys. Rev. E 82, 032401 (2010).

[138] R. Gomez-Solano, A. Petrosyan, S. Ciliberto, and C. Maes, Fluctuations and Response in a Non-Equilibrium MicronSized System, J. Stat. Mech. (2011) P01008.

[139] M. Baiesi, S. Ciliberto, G. Falasco, and C. Yolcu, Thermal Response of Nonequilibrium RC Circuits, Phys. Rev. E 94, 022144 (2016). 
[140] C. Yolcu, A. Bérut, G. Falasco, A. Petrosyan, S. Ciliberto, and M. Baiesi, A General Fluctuation Response Relation for Noise Variations and Its Application to Driven Hydrodynamic Experiments, J. Stat. Phys. 167, 29 (2017).

[141] E. Lutz and S. Ciliberto, Information: From Maxwell's Demon to Landauer's Eraser, Phys. Today 68, No. 9, 30 (2015).

[142] J. M. R. Parrondo, J. M. Horowitz, and T. Sagawa, Thermodynamics of Information, Nat. Phys. 11, 131 (2015).

[143] S. Toyabe, T. Sagawa, M. Ueda, M. Muneyuki, and M. Sano, Experimental Demonstration of Informationto-Energy Conversion and Validation of the Generalized Jarzynski Equality, Nat. Phys. 6, 988 (2010).

[144] T. Sagawa and M. Ueda, Generalized Jarzynski Equality under Nonequilibrium Feedback Control, Phys. Rev. Lett. 104, 090602 (2010).

[145] T. Sagawa and M. Ueda, Minimum Energy Cost for Thermodynamic Information Processing: Measurement and Information Erasure, Phys. Rev. Lett. 102, 250602 (2009).

[146] J. V. Koski, V. F. Maisi, T. Sagawa, and J. P. Pekola, Experimental Observation of the Role of Mutual Information in the Nonequilibrium Dynamics of a Maxwell Demon, Phys. Rev. Lett. 113, 030601 (2014).

[147] J. V. Koski, A. Kutvonen, I. M. Khaymovich, T. AlaNissila, and J. P. Pekola, On-Chip Maxwell's Demon as an Information-Powered Refrigerator, Phys. Rev. Lett. 115, 260602 (2015).

[148] J. V. Koski and J. P. Pekola, Maxwell's Demons Realized in Electronic Circuits, C.R. Phys. 17, 1130 (2016).

[149] J. M. Horowitz and M. Esposito, Thermodynamics with Continuous Information Flow, Phys. Rev. X 4, 031015 (2014).

[150] A. Bérut, A. Arakelyan, A. Petrosyan, S. Ciliberto, R. Dillenschneider, and E. Lutz, Experimental Verification of Landauer's Principle Linking Information and Thermodynamics, Nature (London) 483, 187 (2012).

[151] A. Bérut, A. Petrosyan, and S. Ciliberto, Detailed Jarzynski Equality Applied to a Logically Irreversible Procedure, Europhys. Lett. 103, 60002 (2013).

[152] M. Gavrilov and J. Bechhoefer, Arbitrarily Slow, NonQuasistatic, Isothermal Transformations, Europhys. Lett. 114, 50002 (2016).

[153] E. Aurell, K. Gawedzki, C. Meja-Monasterio, R. Mohayaee, and P. Muratore-Ginanneschi, Refined Second Law of Thermodynamics for Fast Random Processes, J. Stat. Phys. 147, 487 (2012).

[154] S. Vaikuntanathan and C. Jarzynski, Dissipation and Lag in Irreversible Processes, Europhys. Lett. 87, 60005 (2009).

[155] M. Gavrilov, R. Chetrite, and J. Bechhoefer, Direct Measurement of Nonequilibrium System Entropy Is Consistent with Gibbs-Shannon Form, arXiv:1703.07601.

[156] J. V. Koski, V. F. Maisi, J. P. Pekola, and D. V. Averin, Experimental Realization of a Szilard Engine with a Single Electron, Proc. Natl. Acad. Sci. U.S.A. 111, 13786 (2014).

[157] E. Roldàn, I. A. Martinez, J. M. R. Parrondo, and D. Petrov, Universal Features in the Energetics of Symmetry Breaking, Nat. Phys. 10, 457 (2014).
[158] Y. Jun, M. Gavrilov, and J. Bechhoefer, High-Precision Test of Landauer's Principle in a Feedback Trap, Phys. Rev. Lett. 113, 190601 (2014).

[159] J. Hong, B. Lambson, S. Dhuey, and J. Bokor, Experimental Test of Landauer's Principle in Single-Bit Operations on Nanomagnetic Memory Bits, Sci. Adv. 2, e1501492 (2016).

[160] M. Gavrilov and J. Bechhoefer, Erasure without Work in an Asymmetric, Double-Well Potential, Phys. Rev. Lett. 117, 200601 (2016).

[161] A. C. Barato, D. Hartich, and U. Seifert, Efficiency of Cellular Information Processing, New J. Phys. 16, 103024 (2014).

[162] S. Ito and T. Sagawa, Maxwell's Demon in Biochemical Signal Transduction with Feedback Loop, Nat. Commun. 6, 7498 (2015).

[163] A. C. Barato and U. Seifert, Cost and Precision of Brownian Clocks, Phys. Rev. X 6, 041053 (2016).

[164] T. E. Ouldridge, C. C. Govern, and P. R. ten Wolde, Thermodynamics of Computational Copying in Biochemical Systems, Phys. Rev. X 7, 021004 (2017).

[165] N. Garnier and S. Ciliberto, Nonequilibrium Fluctuations in a Resistor, Phys. Rev. E 71, 060101 (2005).

[166] K. Hayashi, H. Ueno, R. Lino, and H. Noji, Fluctuation Theorem Applied to $\mathrm{F}_{1}$-ATPase, Phys. Rev. Lett. 104, 218103 (2010).

[167] E. Zimmermann and U. Seifert, Effective Rates from Thermodynamically Consistent Coarse-Graining of Models for Molecular Motors with Probe Particles, Phys. Rev. E 91, 022709 (2015).

[168] G. Bulnes Cuetara, M. Esposito, and M. Gaspard, Fluctuation Theorems for Capacitively Coupled Electronic Currents, Phys. Rev. B 84, 165114 (2011).

[169] Y. Utsumi, D. S. Golubev, M. Marthaler, K. Saito, T. Fujisawa, and G. Schon, Bidirectional Single-Electron Counting and the Fluctuation Theorem, Phys. Rev. B 81, 125331 (2010).

[170] B. Kung, C. Rossler, M. Beck, M. Marthaler, D. S. Golubev, Y. Utsumi, T. Ihn, and K. Ensslin, Irreversibility on the Level of Single-Electron Tunneling, Phys. Rev. X 2, 011001 (2012).

[171] J. Mehl, B. Lander, C. Bechinger, V. Blickle, and U. Seifert, Role of Hidden Slow Degrees of Freedom in the Fluctuation Theorem, Phys. Rev. Lett. 108, 220601 (2012).

[172] A. Alemany, M. Ribezzi-Crivellari, and F. Ritort, From Free Energy Measurements to Thermodynamic Inference in Nonequilibrium Small Systems, New J. Phys. 17, 075009 (2015).

[173] E. Dieterich, J. Camunas-Solert, M. Ribezzi-Crivellari, U. Seifert, and F. Ritort, Single-Molecule Measurement of the Effective Temperature in Non-Equilibrium Steady States, Nat. Phys. 11, 971 (2015).

[174] P. Strasberg, G. Schaller, T. Brandes, and M. Esposito, Quantum and Information Thermodynamics: A Unifying Framework Based on Repeated Interactions, Phys. Rev. X 7, 021003 (2017). 